\title{
Optimal Multiplicative Generalized Linear Search Plan for a Discrete Random Walker
}

\author{
Abd-Elmoneim Anwar Mohamed and Mohamed Abd Allah El-Hadidy \\ Department of Mathematics, Faculty of Science, Tanta University, Tanta 31527, Egypt \\ Correspondence should be addressed to Mohamed Abd Allah El-Hadidy; melhadidi@eun.eg
}

Received 18 February 2013; Accepted 14 June 2013

Academic Editor: Bijaya Panigrahi

Copyright ( 2013 A.-E. A. Mohamed and M. A. A. El-Hadidy. This is an open access article distributed under the Creative Commons Attribution License, which permits unrestricted use, distribution, and reproduction in any medium, provided the original work is properly cited.

\begin{abstract}
This paper formulates a search model that gives the optimal search plan for the problem of finding a discrete random walk target in minimum time. The target moves through one of $n$-disjoint real lines in $\mathbb{R}^{n}$ : we have $n$-searchers starting the searching process for the target from any point rather than the origin. We find the conditions that make the expected value of the first meeting time between one of the searchers and the target finite. Furthermore, we show the existence of the optimal search plan that minimizes the expected value of the first meeting time and find it. The effectiveness of this model is illustrated using numerical example.
\end{abstract}

\section{Introduction}

The search problem for a randomly moving target has a remarkable importance in our life due to its great applicability. This problem is very interesting because it may arise in many real world situations such as searching for randomly moving persons or targets on roads. That mathematical analysis collected all the researches derived from searching applications of the II World War. They solved with beauty two complementary objectives of the search: find the target with (1) the smallest cost and (2) in minimum time. Readers are referred to Koopman [1] for the early works and to Benkoski et al. [2] and Frost and Stone [3] for a more recent survey.

In the linear search problem, the target moves on the real line according to a known random process, and its initial position is given by the value of a random variable $X_{0}$ which has known probability distribution function. A searcher starts looking for the target at a point $H_{0}\left(\left|H_{0}\right|<\right.$ $\infty)$. The searcher moves continuously along the line in both directions of the starting point $H_{0}$ until the target is met. The searcher would change its direction at suitable points many times before meeting its goal. Thus, we consider that the path length which we represented is the cost of the search. The aim of the searcher is to minimize $E_{\tau_{\phi}}$, that is, the expected value of the first meeting time $\tau_{\phi}$ between the searcher and the target. The problem is to find a search plan $\phi(t)$, such that $E_{\tau_{\phi}}<\infty$; in this case, we call that $\phi(t)$ is a finite search plan and if $E_{\tau_{\phi^{*}}}<E_{\tau_{\phi}}$ for all $\phi(t) \in \Phi(t)$, where $\Phi(t)$ terms to the class of all search plans then we call that $\phi^{*}(t)$ is an optimal search plan.

MC Cabe [4] found a finite search plan for a onedimensional random walk target when the searcher starts the search from the origin, and the initial position of the target has a standard normal distribution. Mohamed [5] discussed the existence of a finite search plan for a one-dimensional random walk target in general case which means that the search may start from any point on the real line, and the initial position of the target has any distribution. El-Rayes and Mohamed [6] have shown the existence of a search plan which minimizes the expected value of the first meeting time between the searcher and the randomly moving target with imposed conditions. Fristedt and Heath [7] derived the conditions for optimal search path which minimizes the cost of effort of finding a randomly moving target on the real line. Ohsumi [8] presented an optimal search plan for a target 
moving with Markov process along one of $K$-nonintersecting arcs to a safe destination within a time limit, where the target starts at a safe base and tries to pass. El-Rayes et al. [9] illustrated this problem when the target moves on the real line with a Brownian motion, and the searcher starts the search from the origin. Recently, Mohamed et al. [10] discussed this problem for a Brownian target motion on one of $n$-intersected real lines in which any information of the target position is not available to the searchers all the time. Mohamed et al. formulate a search model and find the conditions under which the expected value of the first meeting time between one of the searchers and the target is finite. Furthermore, they showed the existence of the optimal search plan that minimizes the expected value of the first meeting time and found it.

On the other hand, when the target is located somewhere on the real line according to a known probability distribution, the searcher searches for it with known velocity and tries to find it in minimal expected time. It is assumed that the searcher can change the direction of its motion without any loss of time. The target can be detected only if the searcher reaches the target. In an earlier work, this problem has been studied extensively in many variations, mostly by Beck et al. [11-17], Franck [18], Rousseeuw [19], Reyniers [20, 21], and Balkhi $[22,23]$.

Furthermore, Mohamed et al. [24, 25] have got more interesting results when they studied this problem to find a randomly located target in the plane. The target has symmetric or asymmetric distribution and with less information about this target available to the searchers. More recently, Mohamed and El-Hadidy [26] studied this problem when the target moves with parabolic spiral in the plane and starts its motion from a random point. Also, Mohamed and El-Hadidy [27] disscussed this problem in the plane when the target moves randomly with conditionally deterministic motion.

Some problems of search may impose using more than one searcher such as when we search for a valuable target (e.g., person lost on one of $n$-disjoint roads) or search for a serious target (e.g., a car filled with explosives which moves randomly in one of $n$-disjoint roads). Thus, the main contributions of this paper center around studying the problem of searching for a one-dimensional random walker target that is moving on one of a system of $n$-disjoint continuous real lines in $\mathbb{R}^{n}$ (i.e., not intersected continuous real lines in the $n$-space). The problem that is studied here is very interesting where we argue to give the conditions on a strategy (or trajectories) of $n$-searchers, one on each line, that make the expected value of the first meeting time between one of the searchers and the target be finite and minimum. In this problem there exists a complication of such analysis. This complication is due to the fact that the searchers do not know the initial position of the target but only know its probability distribution. Otherwise, the problem would be reduced to determine the strategy of just one searcher on the same target's line. This problem is already tackled in $[6,7]$. This work focuses on the necessary conditions for the existence of finite and optimal search plan that finds a random walker target.

The optimal search plan that is proposed here shows that the special structure of the search problem can be exploited

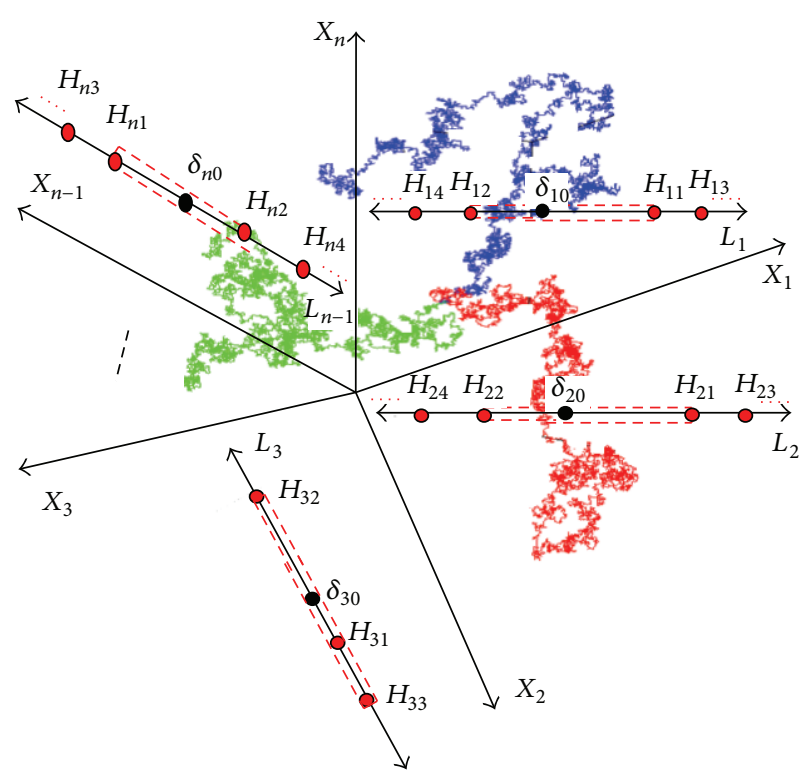

FIgURE 1: The search plan $\phi(t)=\left(\phi_{1}(t), \phi_{2}(t), \ldots, \phi_{n}(t)\right)$ of the searchers $S_{i}, i=1,2, \ldots, n$.

to obtain the efficient solution. For example, the search plan for a criminal drunk leaves its cache and walks up and down through one of $n$-disjoint streets, totally disoriented.

This paper is organized as follows. In Section 2 we formulate the problem. We display some properties that the search model should satisfy in Section 3. The search plan and the conditions that make the expected value of the first meeting time between one of the searchers and the target be finite is discussed in Section 4. The existence of optimal search plan that minimizes the expected value of the first meeting time is presented in Section 5. The optimal search plan is studied in Section 6. In Section 7 we illustrate the effectiveness of this model using numerical example. Finally, the paper concludes with a discussion of the results and directions for future research.

\section{Problem Description}

The problem under study can be formally described as follows. We have $n$-searchers $S_{i}, i=1,2, \ldots, n$, that start the searching process from any point rather than the origin of the line $L_{i}, i=1,2, \ldots, n$, respectively, as in Figure 1. Each of the searchers moves continuously along its line in both directions of the starting point. The searcher $S_{i}$ would conduct its search in the following manner. Start at $\delta_{i 0}$ and go to the left (right) as far as $H_{i 1}$. Then, turn back to explore the right (left) part of $\delta_{i 0}$ as far as $H_{i 2}$. Retrace the steps again to explore the left (right) part of $H_{i 1}$ as far as $H_{i 3}$ and so forth. In this paper, we need to determine $H_{i j}, i=1,2, \ldots, n, j=1,2, \ldots$, that minimize the first meeting time between one of the searchers and the moving target. 
Let $I$ be a set of integer numbers and $I^{+}$a nonnegative part of $I$. We also assume that $\left\{X_{i j}\right\}_{j \geq 1}$ are sequences of independent identically distributed random variables in $L_{i}, i=1,2, \ldots, n$, respectively. In addition, we let a value of " -1 " indicate a step to the left and a value " 1 " a step to the right, so that, for any $j \geq 1$, we have the $n$ dimensional probability vectors, $\left[P\left(X_{1 j}=1\right)=p_{1}, P\left(X_{2 j}=\right.\right.$ $\left.1)=p_{2}, \ldots, P\left(X_{n j}=1\right)=p_{n}\right]$ and $\left[P\left(X_{1 j}=-1\right)=\right.$ $\left.q_{1}, P\left(X_{2 j}=-1\right)=q_{2}, \ldots, P\left(X_{n j}=-1\right)=q_{n}\right]$, where $\left[p_{1}, p_{2}, \ldots, p_{n}\right]+\left[q_{1}, q_{2}, \ldots, q_{n}\right]=[1,1, \ldots, 1]$.

Supposing that, for $t>0, t \in I^{+}, W(t)=$ $\sum_{j=1}^{t} X_{i j}, W(0)=0$ and $X_{0}$ is a random variable that represent the target initial position, valued in $2 I$ or $(2 I+1)$ and independent of $W(t), t \geq 0$; if $\xi_{i}>0$ and $X_{i j}$ such that $0 \leq K_{i 1}=\left(\xi_{i}+x_{i}\right) / 2 \leq \xi_{i}$, where $K_{i 1}$ is an integer, then

$$
\begin{aligned}
& P\left(W\left(\xi_{i}\right)=K_{i 1}\right) \\
& = \begin{cases}\left(\begin{array}{c}
\xi_{i} \\
K_{i 1}
\end{array}\right) p_{i}^{K_{i 1}} q_{i}^{\xi_{i}-K_{i 1}}, & \\
0, & \text { if } K_{i 1} \text { does not exist. }\end{cases}
\end{aligned}
$$

The target is assumed to move randomly on one of $n$ disjoint real lines according to the process $\left\{W(t), t \in I^{+}\right\}$, where $I^{+}$is the set of positive integer numbers and $W(t)$ is a one-dimensional random walk motion. The initial position of the target is unknown but the searchers know its probability distribution (i.e., the probability distribution of the target is given at time 0$)$ and the process $\left\{W(t), t \in I^{+}\right\}$which controls the target's motion.

Figure 1 gives an illustration of the search plan that the $n$ searchers $S_{i}, i=1,2, \ldots, n$, follow it. Moreover, it is to be noted that this search plan $\phi(t)=\left(\phi_{1}(t), \phi_{2}(t), \ldots, \phi_{n}(t)\right)$ is a combination of continuous functions $\phi_{i}(t)$ with speed $v_{i}$ and given by $\phi_{i}(t): I^{+} \rightarrow I, i=1,2, \ldots, n$, such that

$$
\begin{array}{r}
\left|\phi_{i}\left(t_{1}\right)-\phi_{i}\left(t_{2}\right)\right|<v_{i}\left|t_{1}-t_{2}\right| \quad \forall t_{1}, t_{2} \in I^{+}, \\
\phi_{i}(0)=0, \quad i=1,2, \ldots, n .
\end{array}
$$

The first meeting time $\tau_{\phi}$ is a random variable valued in $I^{+}$, and it is defined by

$$
\tau_{\phi}=\left\{\begin{aligned}
& \inf \left\{t: \text { either one of } \phi_{i}(t)=X_{0}+W(t),\right. \\
& \quadi=1,2, \ldots, n\} \\
& \infty, \quad \text { if the set is empty, }
\end{aligned}\right.
$$

where $X_{0} \in I$, that is, a random variable independent of $W(t)$, and it represents the initial position of the target. We suppose that $X_{0}=X_{i}$ if the target moves on $L_{i}, i=1,2, \ldots, n$. Also assume that the set of all search plans of the searchers $S_{i}$ with speeds $v_{i}, i=1,2, \ldots, n$, respectively, satisfying condition (2) be $\Phi_{v_{i}}(t), i=1,2, \ldots, n$. The problem is to find a search plan $\phi(t)=\left(\phi_{1}(t), \phi_{2}(t), \ldots, \phi_{n}(t)\right) \in \Phi(t)$ for the $n$-searchers such that $E_{\tau_{\phi}}<\infty$, where $E_{\tau_{\phi}}$ is the expected value of the first meeting time between one of the searchers and the target, and $\Phi(t)=\left\{\left(\phi_{1}(t), \phi_{2}(t), \ldots, \phi_{n}(t)\right) ; \phi_{i}(t) \in \Phi_{v_{i}}(t), i=\right.$ $1,2, \ldots, n\}$, is called a class of all sets of the search plans.
Assuming that $\vartheta_{i}, \lambda_{i}, i=1,2, \ldots, n$, are positive numbers such that $\vartheta_{i}>1, C_{i}=\left(\vartheta_{i}-1\right) /\left(\vartheta_{i}+1\right)>\left|p_{i}-q_{i}\right|, \lambda_{i}=$ $b \omega_{i}, b=1,2, \ldots$, and $\varrho_{i}\left(1-C_{i}\right) / 2$ is positive number also. In this problem, we assume that $v_{i}=1, i=1,2, \ldots, n$. In addition, we define the sequences $\left\{G_{i j}\right\},\left\{d_{i j}\right\}$, and $\left\{H_{i j}\right\}, i=$ $1,2, \ldots, n, j=1,2, \ldots$ by $G_{i j}=\lambda_{i}\left(\vartheta_{i}^{j}-1\right), d_{i j}=$ $(-1)^{j+1} C_{i}\left[G_{i j}+1+(-1)^{j+1}\right]$, and $H_{i j}=d_{i j}+\delta_{i 0}$. We also use the notations $\psi_{i}\left(G_{i(2 j+1)}\right)=W\left(G_{i(2 j+1)}\right)-K_{i 1}\left(G_{i(2 j+1)}\right)$, $\widehat{\psi}_{i}\left(G_{i(2 j)}\right)=W\left(G_{i(2 j)}\right)+K_{i 2}\left(G_{i(2 j)}\right)$, where $K_{i 1}\left(G_{i(2 j+1)}\right)=$ $C_{i} G_{i(2 j+1)}, K_{i 2}\left(G_{i(2 j)}\right)=C_{i} G_{i(2 j)}$ which are positive functions and the following remark.

Remark 1. If $0<\varkappa_{\S}<1, \S=1,2, \ldots, \kappa$, then $\prod_{\S=1}^{\kappa} \varkappa_{\S}<$ $\sum_{\S=1}^{\kappa} \varkappa_{\S}$.

There are known probability measures $\gamma_{i}, i=1,2, \ldots, n$, such that $\gamma_{1}+\gamma_{2}+\cdots+\gamma_{n}=1$ on $L_{1} \cup L_{2} \cup \cdots \cup L_{n}$. And, they describe the location of the target, where $\gamma_{i}$ is induced by the position of the target on $L_{i}$.

The objective is to obtain the conditions that make the search plan $\phi(t)=\left(\phi_{1}(t), \phi_{2}(t), \ldots, \phi_{n}(t)\right)$ be finite (i.e., $E_{\tau_{\phi}}<$ $\infty)$. We also need to show the existence of the optimal search plan $\phi^{*}(t)=\left(\phi_{1}^{*}(t), \phi_{2}^{*}(t), \ldots, \phi_{n}^{*}(t)\right)$ and find it, that is, to give the minimum expected value of the first meeting time.

\section{Properties of the Search Model}

In this section we start to discuss some properties that the search model for a one-dimensional random walk target should satisfy.

The searcher $S_{i}$ would conduct its search as in the above manner that is detailed in Section 2. Consequently, for any line $L_{i}, i=1,2, \ldots, n, t \in I^{+}$, we have the following.

Case 1. If we consider that $\mathbb{Q}$ is a set of positive even numbers such that $\mathbb{Q}=\{2,4,6, \ldots, \widehat{n}\}$, for $\mathscr{F} \in \mathbb{Q}, j \in I^{+}$, then we have

$$
\begin{aligned}
\cdots & <H_{i(j+2)}<0<H_{i j}<H_{i(j-2)}<\cdots<H_{i 2}<\delta_{i 0} \\
& <H_{i 1}<H_{i 3}<\cdots ;
\end{aligned}
$$

for any $t \in I^{+}$, if $G_{i(2 j-1)} \leq t \leq G_{i(2 j)}, \quad 1 \leq j \leq \mathscr{F} / 2$, then

$$
\begin{array}{r}
\phi_{i}(t)=H_{i(2 j+1)}-\delta_{i 0}-\left[t-G_{i(2 j-1)}\right] \\
\text { if } G_{i j} \leq t \leq G_{i(j+1)}, j \geq \mathscr{F}+1 \text {, then } \\
\phi_{i}(t)=H_{i j}+(-1)^{j}\left[t-G_{i j}\right] \\
\text { if } G_{i(2 j)} \leq t \leq G_{i(2 j+1)}, 1 \leq j \leq \mathscr{F} / 2 \text {, then } \\
\phi_{i}(t)=\delta_{i 0}-H_{i(2 j)}+\left[t-G_{i(2 j)}\right] .
\end{array}
$$

Case 2. Also, if $O$ is a set of positive odd numbers such that $O=\{1,3,5, \ldots, \widehat{m}\}$, for $\mathscr{F} \in O, j \in I^{+}$, then we have

$$
\begin{aligned}
\cdots & <H_{i 2}<\delta_{i 0}<H_{i 1}<H_{i 3}<\cdots<H_{i(j-2)}<H_{i j} \\
& <0<H_{i(j+2)}<\cdots ;
\end{aligned}
$$


for any $t \in I^{+}$, if $G_{i(2 j-1)} \leq t \leq G_{i(2 j)}, 1 \leq j \leq(\mathscr{F}+1) / 2$, then

$$
\phi_{i}(t)=\delta_{i 0}-H_{i(2 j-1)}-\left[t-G_{i(2 j-1)}\right] ;
$$

if $G_{i j} \leq t \leq G_{i(j+1)}, j \geq \mathscr{F}+1$, then

$$
\phi_{i}(t)=H_{i j}+(-1)^{j}\left[t-G_{i j}\right] ;
$$

if $G_{i(2 j)} \leq t \leq G_{i(2 j+1)}, 1 \leq j \leq(\mathscr{F}-1) / 2$, then

$$
\phi_{i}(t)=H_{i(2 j)}-\delta_{i 0}+\left[t-G_{i(2 j)}\right] .
$$

It is clear that the search path of $S_{i}$ depends on $\lambda_{i}, \vartheta_{i}$ and $j \in I^{+}$. Since $X_{0} \in I$ then the first meeting time is done at $x_{i} \in I$ where $x_{i}$ is an integer number. For this reason we will prove the conditions (properties) that make $S_{i}$ meet the target at $x_{i}$ as in the following theorems.

Theorem 2. If $C_{i}$ is a rational number different from 1 to -1 and, for all $\xi_{i} \geq 1, V\left(\xi_{i}\right)=\left(W\left(\xi_{i} \vartheta_{i}\right)-C_{i} \xi_{i} \vartheta_{i}\right) / 2$ then

(i) there exists a sequence $\left\{Y_{i j}\right\}_{j \geq 1}$ of independent identically distributed random variables such that $V\left(\xi_{i}\right)=$ $\sum_{j=1}^{\Gamma} Y_{i j}$, and the distribution of $Y_{i j}$ is concentrated on the integers $E\left(Y_{i j}\right)=\left(\vartheta_{i} / 2\right)\left[E\left(X_{i j}\right)-C_{i}\right]$ and the probability vector $\left[p\left(Y_{1 j}=\mathscr{F}\right)>0, p\left(Y_{2 j}=\mathscr{F}\right)>\right.$ $\left.0, \ldots, p\left(Y_{n j}=\mathscr{F}\right)>0\right]$, if and only if $-\vartheta_{i}\left(1+C_{i}\right) / 2 \leq$ $\mathscr{F} \leq \vartheta_{i}\left(1-C_{i}\right) / 2$.

(ii) The probability vector, $\left[p\left(V\left(\xi_{1}\right)=x_{1}\right)>0, p\left(V\left(\xi_{2}\right)=\right.\right.$ $\left.x_{2}\right)>0, \ldots, p\left(V\left(\xi_{n}\right)=x_{n}\right)>0$, holds if and only if $x_{i}$ is an integer such that $-\xi_{i} \vartheta_{i}\left(1+C_{i}\right) / 2 \leq x_{i} \leq$ $\xi_{i} \vartheta_{i}\left(1-C_{i}\right) / 2$.

(iii) If $C_{i} \neq p_{i}-q_{i}$ then there exist constants $r_{i 1}$ and $r_{i 2}$ depending on $C_{i}, p_{i}$ such that, for any $x_{i} \in \mathbb{R}$, where $\mathbb{R}$ is the set of real numbers, if $\xi_{i}>r_{i 1} x_{i}+r_{i 2}$ and if $x_{i} \geq 0$ we have $\left[p\left(0 \leq V\left(\xi_{1}+1\right) \leq x_{1}\right), p\left(0 \leq V\left(\xi_{2}+1\right) \leq\right.\right.$ $\left.\left.x_{2}\right), \ldots, p\left(0 \leq V\left(\xi_{n}+1\right) \leq x_{n}\right)\right] \leq\left[p\left(0 \leq V\left(\xi_{1}\right) \leq\right.\right.$ $\left.\left.x_{1}\right), p\left(0 \leq V\left(\xi_{2}\right) \leq x_{2}\right), \ldots, p\left(0 \leq V\left(\xi_{n}\right) \leq x_{n}\right)\right]$, and if $x_{i}<0$ we have, $\left[p\left(x_{1} \leq V\left(\xi_{1}+1\right)<0\right), p\left(x_{2} \leq\right.\right.$ $\left.\left.V\left(\xi_{2}+1\right)<0\right), \ldots, p\left(x_{n} \leq V\left(\xi_{n}+1\right)<0\right)\right] \leq\left[p\left(x_{1} \leq\right.\right.$ $\left.V\left(\xi_{1}\right)<0\right), p\left(x_{2} \leq V\left(\xi_{2}\right)<0\right), \ldots, p\left(x_{n} \leq V\left(\xi_{n}\right)<\right.$ $0)]$.

Proof. (i) Define $Y_{i j}=\sum_{\mathscr{F}=1}^{\vartheta_{i}}\left(\left(X_{i\left(\mathscr{F}+(j-1) \vartheta_{i}\right)}-C_{i}\right) / 2\right), j \geq 1$, and $\left[p\left(Y_{1 j}=x_{1}\right), p\left(Y_{2 j}=x_{2}\right), \ldots, p\left(Y_{n j}=x_{n}\right)\right]=$ $\left[p\left(W\left(\vartheta_{1}\right)\right)=2 x_{1}+\vartheta_{1} C_{1}, p\left(W\left(\vartheta_{2}\right)\right)=2 x_{2}+\vartheta_{2} C_{2}, \ldots\right.$, $\left.p\left(W\left(\vartheta_{n}\right)\right)=2 x_{n}+\vartheta_{n} C_{n}\right]$.

Consequently, if $\left[p\left(Y_{1 j}=x_{1}\right)>0, p\left(Y_{2 j}=x_{2}\right)>\right.$ $0, \ldots, p\left(Y_{n j}=x_{n}\right)>0$ ], then from (1) we have $x_{i}+\vartheta_{i}(1+$ $\left.C_{i}\right) / 2$ that is an integer, and since $x_{i}$ is an integer also then we have $0 \leq x_{i}+\vartheta_{i}\left(1+C_{i}\right) / 2 \leq \vartheta_{i}$. Therefore, $-\vartheta_{i}\left(1+C_{i}\right) / 2 \leq$ $x_{i} \leq \vartheta_{i}\left(1-C_{i}\right) / 2$. If $x_{i}=\mathscr{F}$ then we have $\left[p\left(Y_{1 j}=\mathscr{F}\right)>\right.$ $\left.0, p\left(Y_{2 j}=\mathscr{F}\right)>0, \ldots, p\left(Y_{n j}=\mathscr{F}\right)>0\right]$, if and only if $-\vartheta_{i}\left(1+C_{i}\right) / 2 \leq \mathscr{F} \leq \vartheta_{i}\left(1-C_{i}\right) / 2$. In addition, by using (1), $E\left(\sum_{\mathscr{F}=1}^{\vartheta_{i}}\left(\left(X_{i\left(\mathscr{F}+(j-1) \vartheta_{i}\right)}-C_{i}\right) / 2\right)\right)=\left(\vartheta_{i} / 2\right)\left[E\left(X_{i j}\right)-C_{i}\right]$ is proved. (ii) Since $V\left(\xi_{i}\right)=\left(W\left(\xi_{i} \vartheta_{i}\right)-C_{i} \xi_{i} \vartheta_{i}\right) / 2$ then we have $\left[p\left(V\left(\xi_{1}\right)=x_{1}\right), p\left(V\left(\xi_{2}\right)=x_{2}\right), \ldots, p\left(V\left(\xi_{n}\right)=x_{n}\right)\right]=$ $\left[p\left(\left(W\left(\xi_{1} \vartheta_{1}\right)-C_{1} \xi_{1} \vartheta_{1}\right) / 2=x_{1}\right), p\left(\left(W\left(\xi_{2} \vartheta_{2}\right)-C_{2} \xi_{2} \vartheta_{2}\right) / 2=\right.\right.$ $\left.\left.x_{2}\right), \ldots, p\left(\left(W\left(\xi_{n} \vartheta_{n}\right)-C_{n} \xi_{n} \vartheta_{n}\right) / 2=x_{n}\right)\right]=\left[p\left(W\left(\xi_{1} \vartheta_{1}\right)\right)=\right.$ $\left.\left.2 x_{1}+C_{1} \xi_{1} \vartheta_{1}\right), p\left(W\left(\xi_{2} \vartheta_{2}\right)\right)=2 x_{2}+C_{2} \xi_{2} \vartheta_{2}\right), \ldots, p\left(W\left(\xi_{n} \vartheta_{n}\right)\right)=$ $\left.\left.2 x_{n}+C_{n} \xi_{n} \vartheta_{n}\right)\right]$, and using (1) the prove is completed.

(iii) By using (ii), if $x_{i} \geq 0$ then we have $\left[p\left(0 \leq V\left(\xi_{1}\right) \leq\right.\right.$ $\left.\left.x_{1}\right), p\left(0 \leq V\left(\xi_{2}\right) \leq x_{2}\right), \ldots, p\left(0 \leq V\left(\xi_{n}\right) \leq x_{n}\right)\right]=$ $\left[\sum_{j=0}^{\left[x_{1}\right]} p\left(V\left(\xi_{1}\right)=j\right), \sum_{j=0}^{\left[x_{2}\right]} p\left(V\left(\xi_{2}\right)=j\right), \ldots, \sum_{j=0}^{\left[x_{n}\right]} p\left(V\left(\xi_{n}\right)=\right.\right.$ $j)$ ], and if $x_{i}<0$ we get [ $p\left(x_{1} \leq V\left(\xi_{1}+1\right)<0\right), p\left(x_{2} \leq V\left(\xi_{2}+\right.\right.$ $\left.1)<0), \ldots, p\left(x_{n} \leq V\left(\xi_{n}+1\right)<0\right)\right]=\left[\sum_{j=\left[x_{1}\right]}^{0} p\left(V\left(\xi_{1}\right)=\right.\right.$ $\left.j), \sum_{j=\left[x_{2}\right]}^{0} p\left(V\left(\xi_{2}\right)=j\right), \ldots, \sum_{j=\left[x_{n}\right]}^{0} p\left(V\left(\xi_{n}\right)=j\right)\right]$, where $\left[x_{i}\right]$ means the greatest integer less than or equal to $x_{i}, i=$ $1,2, \ldots, n$. It is sufficient to show that

$$
\begin{array}{r}
{\left[p\left(V\left(\xi_{1}+1\right)=\mathscr{F}\right), p\left(V\left(\xi_{2}+1\right)=\mathscr{F}\right), \ldots,\right.} \\
\left.p\left(V\left(\xi_{n}+1\right)=\mathscr{F}\right)\right] \\
\leq\left[p\left(V\left(\xi_{1}\right)=\mathscr{F}\right), p\left(V\left(\xi_{2}\right)=\mathscr{F}\right), \ldots,\right. \\
\left.p\left(V\left(\xi_{n}\right)=\mathscr{F}\right)\right] \quad \text { if } \xi_{i}>r_{i 1} x_{i}+r_{i 2} .
\end{array}
$$

We have the following cases.

Case (a). If $C_{i}<-1$, then, from (ii), $\left[p\left(V\left(\xi_{1}\right)=\mathscr{F}\right)>\right.$ $0, p\left(V\left(\xi_{2}\right)=\mathscr{F}\right)>0, \ldots, p\left(V\left(\xi_{n}\right)=\mathscr{F}\right)>0$ ], if and only if $2 \mathscr{F} / \vartheta_{i}\left(1-C_{i}\right) \leq \xi_{i} \leq-2 \mathscr{F} / \vartheta_{i}\left(1+C_{i}\right)$. We take $r_{i 1}=-2 / \mathcal{\vartheta}_{i}\left(1+C_{i}\right), r_{i 2}=0$ and then $\xi_{i}>r_{i 1}|\mathscr{F}| \Rightarrow \xi_{i}>$ $-2 \mathscr{F} / \vartheta_{i}\left(1+C_{i}\right)$ that leads to $\left[p\left(V\left(\xi_{1}\right)=\mathscr{F}\right)=0\right.$, $p\left(V\left(\xi_{2}\right)=\right.$ $\left.\mathscr{F})=0, \ldots, p\left(V\left(\xi_{n}\right)=\mathscr{F}\right)=0\right]$. Consequently, (12) holds.

Case (b). If $C_{i}>1$, hence $\left[p\left(V\left(\xi_{1}\right)=\mathscr{F}\right)>0, p\left(V\left(\xi_{2}\right)=\right.\right.$ $\left.\mathscr{F})>0, \ldots, p\left(V\left(\xi_{n}\right)=\mathscr{F}\right)>0\right]$, if and only if $-2 \mathscr{F} / \vartheta_{i}(1+$ $\left.C_{i}\right) \leq \xi_{i} \leq 2 \mathscr{F} / \vartheta_{i}\left(1-C_{i}\right)$. From (ii) putting $r_{i 1}=-2 / \vartheta_{i}(1-$ $\left.C_{i}\right), r_{i 2}=0$ then $\xi_{i}>r_{i 1}|\mathscr{F}| \Rightarrow \xi_{i}>2 \mathscr{F} / \mathcal{\vartheta}_{i}\left(1-C_{i}\right)$ that leads to $\left[p\left(V\left(\xi_{1}\right)=\mathscr{F}\right)=0, p\left(V\left(\xi_{2}\right)=\mathscr{F}\right)=0, \ldots, p\left(V\left(\xi_{n}\right)=\right.\right.$ $\mathscr{F})=0]$.

Then, (12) holds.

Case (c). If $-1<C_{i}<1$, then let $\alpha_{i}=\left(1-C_{i}\right) / 2, \beta_{i}=$ $1-\alpha_{i}$ and $\Delta_{i}=\left(\alpha_{i} / q_{i}\right)^{\alpha_{i}}\left(\beta_{i} / p_{i}\right)^{\beta_{i}}$, where $\left[p_{1}, p_{2}, \ldots, p_{n}\right]+$ $\left[q_{1}, q_{2}, \ldots, q_{n}\right]=[1,1, \ldots, 1]$. In addition, put $r_{i 2}=1 /\left(\Delta_{i}-\right.$ 1), $r_{i 1}=\Delta_{i} \max \left[\left(1 / \alpha_{i}, 1 / \beta_{i}\right) \vartheta_{i}\left(\Delta_{i}-1\right)\right]$. Since $E\left(X_{i j}\right) \neq C_{i}$ and $0<\alpha_{i}<1$ then $\alpha_{i} \neq q_{i}$ and then $\Delta_{i}>1$. Consequently, $r_{i 1}$ and $r_{i 2}$ are positive and well defined. Assuming that $\xi_{i}>$ $r_{i 1}|\mathscr{F}|$ then $-\beta_{i} \xi_{i} \vartheta_{i}<\mathscr{F}<\alpha_{i} \xi_{i} \vartheta_{i}$; therefore, from (ii), we have $\left[p\left(V\left(\xi_{1}\right)=\mathscr{F}\right)>0, p\left(V\left(\xi_{2}\right)=\mathscr{F}\right)>0, \ldots, p\left(V\left(\xi_{n}\right)=\right.\right.$ $\mathscr{F})>0]$.

It remains to prove that if $\left[p\left(V\left(\xi_{1}\right)=\mathscr{F}\right)>0, p\left(V\left(\xi_{2}\right)=\right.\right.$ $\left.\mathscr{F})>0, \ldots, p\left(V\left(\xi_{n}\right)=\mathscr{F}\right)>0\right]$ and $\xi_{i}>r_{i 1}|\mathscr{F}|+r_{i 2}$ then (2) holds.

Considering that $Z_{i j}=\left(X_{i j}-C_{i}\right) / 2$ then $\left[p\left(Z_{1 j}=\alpha_{1}\right)=\right.$ $\left.p_{1}, p\left(Z_{2 j}=\alpha_{2}\right)=p_{2}, \ldots, p\left(Z_{n j}=\alpha_{n}\right)=p_{n}\right],\left[p\left(Z_{1 j}=\right.\right.$ $\left.\left.-\beta_{1}\right)=q_{1}, p\left(Z_{2 j}=-\beta_{2}\right)=q_{2}, \ldots, p\left(Z_{n j}=-\beta_{n}\right)=q_{n}\right]$, 
and $\left[p\left(V\left(\xi_{1}\right)=\mathscr{F}\right), p\left(V\left(\xi_{2}\right)=\mathscr{F}\right), \ldots, p\left(V\left(\xi_{n}\right)=\mathscr{F}\right)\right]=$ $\left[p\left(\sum_{j=1}^{\xi_{1} \vartheta_{1}} Z_{1 j}=\mathscr{F}\right), p\left(\sum_{j=1}^{\xi_{2} \vartheta_{2}} Z_{2 j}=\mathscr{F}\right), \ldots, p\left(\sum_{j=1}^{\xi_{n} \vartheta_{n}} Z_{n j}=\right.\right.$ $\mathscr{F})$ ]. By using (1) we have

$$
\begin{aligned}
& {\left[\frac{p\left(V\left(\xi_{1}+1\right)=\mathscr{F}\right)}{p\left(V\left(\xi_{1}\right)=\mathscr{F}\right)}, \frac{p\left(V\left(\xi_{2}+1\right)=\mathscr{F}\right)}{p\left(V\left(\xi_{2}\right)=\mathscr{F}\right)}, \ldots,\right.} \\
&\left.\frac{p\left(V\left(\xi_{n}+1\right)=\mathscr{F}\right)}{p\left(V\left(\xi_{n}\right)=\mathscr{F}\right)}\right] \\
&=\left[\prod_{j=1}^{\vartheta_{1} \beta_{1}} \frac{\xi_{1} \vartheta_{1}+j}{\Delta_{1}\left(\xi_{1} \vartheta_{1}+j+\left(j \alpha_{1}+\mathscr{F}\right) / \beta_{1}\right)}\right. \\
& \times \prod_{j=1}^{\vartheta_{1} \alpha_{1}} \frac{\xi_{1} \vartheta_{1}+j+\vartheta_{1} \beta_{1}}{\Delta_{1}\left(\xi_{1} \vartheta_{1}+j+\left(i \beta_{1}-\mathscr{F}\right) / \alpha_{1}\right)}, \\
& \prod_{j=1}^{\vartheta_{2} \beta_{2}} \frac{\xi_{2} \vartheta_{2}+j}{\Delta_{2}\left(\xi_{2} \vartheta_{2}+j+\left(j \alpha_{2}+\mathscr{F}\right) / \beta_{2}\right)} \\
& \times \prod_{j=1}^{\vartheta_{2} \alpha_{2}} \frac{\xi_{2} \vartheta_{2}+j+\vartheta_{2} \beta_{2}}{\Delta_{2}\left(\xi_{2} \vartheta_{2}+j+\left(i \beta_{2}-\mathscr{F}\right) / \alpha_{2}\right)}, \ldots, \\
&\left.\times \prod_{j=1}^{\vartheta_{n} \beta_{n}} \frac{\xi_{n} \vartheta_{n}+j+\vartheta_{n} \beta_{n}}{\Delta_{n}\left(\xi_{n} \vartheta_{n}+j+\left(i \beta_{n}-\mathscr{F}\right) / \alpha_{n}\right)}\right] . \\
& \prod_{j=1}^{\vartheta_{n} \alpha_{n}\left(\xi_{n} \vartheta_{n}+j+\left(j \alpha_{n}+\mathscr{F}\right) / \beta_{n}\right)}
\end{aligned}
$$

Since $\xi_{i}>r_{i 1}|\mathscr{F}|+r_{i 2}$ then every term is strictly less than 1. Consequently, $\left[p\left(V\left(\xi_{1}+1\right)=\mathscr{F}\right), p\left(V\left(\xi_{2}+1\right)=\right.\right.$ $\left.\mathscr{F}), \ldots, p\left(V\left(\xi_{n}+1\right)=\mathscr{F}\right)\right]<\left[p\left(V\left(\xi_{1}\right)=\mathscr{F}\right), p\left(V\left(\xi_{2}\right)=\right.\right.$ $\left.\mathscr{F}), \ldots, p\left(V\left(\xi_{n}\right)=\mathscr{F}\right)\right]$.

Theorem 3. If $E\left(X_{i j}\right)<C_{i}, C_{i} \in \mathbb{R}$ (the set of real numbers), then there exists $\varepsilon_{i}, 0<\varepsilon_{i}<1$, such that $\left[p\left(W\left(\xi_{1}\right)\right), p\left(W\left(\xi_{2}\right)\right), \ldots, p\left(W\left(\xi_{n}\right)\right)\right] \leq\left[\varepsilon_{1}^{\xi_{1}}, \varepsilon_{2}^{\xi_{2}}, \ldots, \varepsilon_{n}^{\xi_{n}}\right]$, for all $\xi_{i}, i=1,2, \ldots, n$.

Proof. For $\zeta_{i}>0,\left[p\left(W\left(\xi_{1}\right) \geq C_{1} \xi_{1}\right), p\left(W\left(\xi_{2}\right) \geq C_{2} \xi_{2}\right), \ldots\right.$, $\left.p\left(W\left(\xi_{n}\right) \geq C_{n} \xi_{n}\right)\right]=\left[p\left(\exp \left\{\zeta_{1}\left(W\left(\xi_{1}\right)-C_{1} \xi_{1}\right)\right\} \geq 1\right), p(\exp \right.$ $\left.\left.\left\{\zeta_{2}\left(W\left(\xi_{2}\right)-C_{2} \xi_{2}\right)\right\} \geq 1\right), \ldots, p\left(\exp \left\{\zeta_{n}\left(W\left(\xi_{n}\right)-C_{n} \xi_{n}\right)\right\} \geq 1\right)\right] \leq$ $\left[E\left(\exp \left\{\zeta_{1}\left(W\left(\xi_{1}\right)-C_{1} \xi_{1}\right)\right\}\right), E\left(\exp \left\{\zeta_{2}\left(W\left(\xi_{2}\right)-C_{2} \xi_{2}\right)\right\}\right), \ldots\right.$, $\left.E\left(\exp \left\{\zeta_{n}\left(W\left(\xi_{n}\right)-C_{n} \xi_{n}\right)\right\}\right)\right]=\left[\left\{f_{1}\left(\zeta_{1}\right)\right\}^{\xi_{1}},\left\{f_{2}\left(\zeta_{2}\right)\right\}^{\xi_{2}}, \ldots\right.$, $\left.\left\{f_{n}\left(\zeta_{n}\right)\right\}^{\xi_{n}}\right]$, where $\left[\left\{f_{1}\left(\zeta_{1}\right)\right\},\left\{f_{2}\left(\zeta_{2}\right)\right\}, \ldots,\left\{f_{n}\left(\zeta_{n}\right)\right\}\right]=[E(\exp$ $\left.\left\{\zeta_{1}\left(X_{1 j}-C_{1}\right)\right\}\right), E\left(\exp \left\{\zeta_{2}\left(X_{2 j}-C_{2}\right)\right\}\right), \ldots, E\left(\exp \left\{\zeta_{n}\left(X_{n j}-\right.\right.\right.$ $\left.\left.\left.\left.C_{n}\right)\right\}\right)\right]=\left[p_{1} \exp \left\{\zeta_{1}\left(1-C_{1}\right)\right\}+q_{1} \exp \left\{\zeta_{1}\left(-1-C_{1}\right)\right\}, p_{2}\right.$ $\exp \left\{\zeta_{2}\left(1-C_{2}\right)\right\}+q_{2} \exp \left\{\zeta_{2}\left(-1-C_{2}\right)\right\}, \ldots, p_{n} \exp$ $\left.\left\{\zeta_{n}\left(1-C_{n}\right)\right\}+q_{n} \exp \left\{\zeta_{n}\left(-1-C_{n}\right)\right\}\right]$; if $E\left(X_{i j}\right)<C_{i}, i=$ $1,2, \ldots, n$, then $\left[\left\{f_{1}^{\prime}(0)\right\},\left\{f_{2}^{\prime}(0)\right\}, \ldots,\left\{f_{n}^{\prime}(0)\right\}\right]<[0,0, \ldots, 0]$, and since $\left[\left\{f_{1}(0)\right\},\left\{f_{2}(0)\right\}, \ldots,\left\{f_{n}(0)\right\}\right]<[1,1, \ldots, 1]$, then $\left[\min _{\zeta_{1}>0}\left\{f_{1}\left(\zeta_{1}\right)\right\}, \min _{\zeta_{2}>0}\left\{f_{2}\left(\zeta_{2}\right)\right\}, \ldots, \min _{\zeta_{n}>0}\left\{f_{n}\left(\zeta_{n}\right)\right\}\right]$ $=\left[\varepsilon_{1}, \varepsilon_{2}, \ldots, \varepsilon_{n}\right]<[1,1, \ldots, 1]$.
By similar arguments if $E\left(X_{i j}\right)>C_{i}, i=1,2, \ldots, n$, then there exist $\varepsilon_{i}<1$ such that $\left[p\left(W\left(\xi_{1}\right)\right), p\left(W\left(\xi_{2}\right)\right), \ldots\right.$, $\left.p\left(W\left(\xi_{n}\right)\right)\right] \leq\left[\varepsilon_{1}^{\xi_{1}}, \varepsilon_{2}^{\xi_{2}}, \ldots, \varepsilon_{n}^{\xi_{n}}\right]$ for all $\xi_{i}, i=1,2, \ldots, n$.

\section{Existence of a Finite Search Plan}

In this section, we find the conditions that make the search plan be finite. In addition, we will discuss that under what these conditions are indeed finite. This is a crucial issue related to the existence of a finite search plan. So, we will provide useful theorems that help us to do it.

Theorem 4. Let $\gamma_{i}$ be the measure defined on $\mathbb{R}$ by $X_{i j}, i=$ $1,2, \ldots, n$, and if $\phi(t)=\left(\phi_{1}(t), \phi_{2}(t), \ldots, \phi_{n}(t)\right)$ is a search plan defined previously, the expectation $E_{\tau_{\phi}}$ is finite if

$$
\begin{aligned}
\int_{-\infty}^{\delta_{i 0}}[ & \sum_{j=1}^{\mathscr{F} / 2} \vartheta_{i}^{2 j} p\left(\psi_{i}\left(G_{i(2 j)}\right)<-x_{i}\right) \\
& \left.+\sum_{j=\mathscr{F} / 2+1}^{\infty} \vartheta_{i}^{2 j} p\left(\tilde{\psi}_{i}\left(G_{i(2 j)}\right) \leq-x_{i}\right)\right] \gamma_{i}\left(d x_{i}\right),
\end{aligned}
$$

$\int_{\delta_{i 0}}^{\infty} \sum_{j=1}^{\infty} \vartheta_{i}^{2 j+1} p\left(\psi_{i}\left(G_{i(2 j+1)}\right)>-x_{i}\right) \psi_{i}\left(d x_{i}\right)$, are finite when $\delta_{i 0}>0$. And if $\delta_{i 0}<0$, then $E_{\tau_{\phi}}$ is finite if

$$
\begin{aligned}
\int_{\delta_{i 0}}^{\infty} & {\left[\sum_{j=(\mathscr{F}+1) / 2}^{\infty} \vartheta_{i}^{2 j+1} p\left(\psi_{i}\left(G_{i(2 j+1)}\right)>-x_{i}\right)\right.} \\
& \left.+\sum_{j=1}^{(\mathscr{F}-1) / 2} \vartheta_{i}^{2 j+1} p\left(\widetilde{\psi}_{i}\left(G_{i(2 j+1)}\right) \leq-x_{i}\right)\right] \gamma_{i}\left(d x_{i}\right),
\end{aligned}
$$

$\int_{-\infty}^{\delta_{i 0}} \sum_{j=1}^{\infty} \vartheta_{i}^{2 j} p\left(\widetilde{\psi}_{i}\left(G_{i(2 j)}\right)<-x_{i}\right) \gamma_{i}\left(d x_{i}\right)$, are finite.

Proof. The hypotheses $X_{i}$ and $\delta_{i 0}$ are valued in $2 I$ or $(2 I+1)$ then $X_{i}+W(t)$ is greater than $\phi_{i}(t)$ until the first meeting; also if $X_{i}$ is smaller than $\delta_{i 0}$ then $X_{i}+W(t)$ is smaller than $\phi_{i}(t)$ until the first meeting. Since $\tau_{\phi_{i}}>t, i=1,2, \ldots, n$, are mutually exclusive events then $p\left(\tau_{\phi_{i}}>t\right)=p\left(\tau_{\phi_{1}}>t\right.$ or $\tau_{\phi_{2}}>t$ or $\cdots$ or $\left.\tau_{\phi_{n}}>t\right)=\sum_{i=1}^{n} p\left(\tau_{\phi_{i}}>t\right)$, and, for any $j \geq 0$ we have

$$
\begin{aligned}
& p\left(\tau_{\phi_{i}}>G_{i(2 j+1)}\right) \\
& \quad \leq \int_{-\infty}^{\delta_{i 0}} p\left(X_{i}+W\left(G_{i(2 j)}\right)<H_{i(2 j)} \mid X_{i}=x_{i}\right) \gamma_{i}\left(d x_{i}\right) \\
& \quad+\int_{\delta_{i 0}}^{\infty} p\left(X_{i}+W\left(G_{i(2 j+1)}\right)>H_{i(2 j+1)} \mid X_{i}=x_{i}\right) \gamma_{i}\left(d x_{i}\right) .
\end{aligned}
$$

Using the notation $\widetilde{\psi}_{i}\left(G_{i(2 j)}\right)=W\left(G_{i(2 j)}\right)+C_{i} G_{i(2 j)}$, we obtain $W\left(G_{i(2 j)}\right)-H_{i(2 j)}<-X_{i}=x_{i}$; then $W\left(G_{i(2 j)}\right)-$ $(-1)^{2 j+1} C_{i}\left[G_{i(2 j)}+1+(-1)^{2 j+1}\right]<-x_{i}$ leads to $W\left(G_{i(2 j)}\right)-$ $(-1) C_{i}\left[G_{i(2 j)}+1+(-1)\right]=W\left(G_{i(2 j)}\right)+C_{i}\left[G_{i(2 j)}\right]=$ 
$\widetilde{\psi}_{i}\left(G_{i(2 j)}\right)<-x_{i}$. Similarly, by using the notation $\psi_{i}\left(G_{i(2 j+1)}\right)=$ $W\left(G_{i(2 j+1)}\right)-C_{i} G_{i(2 j+1)}$, we get $\psi_{i}\left(G_{i(2 j+1)}\right)>-x_{i}$. Consequently,

$$
\begin{aligned}
p\left(\tau_{\phi_{i}}>G_{i(2 j+1)}\right) & \\
\leq & \int_{-\infty}^{\delta_{i 0}} p\left(\widetilde{\psi}_{i}\left(G_{i(2 j)}\right)<-x_{i}\right) \gamma_{i}\left(d x_{i}\right) \\
& +\int_{\delta_{i 0}}^{\infty} p\left(\psi_{i}\left(G_{i(2 j+1)}\right)>-x_{i}\right) \gamma_{i}\left(d x_{i}\right),
\end{aligned}
$$

also,

$$
\begin{aligned}
p\left(\tau_{\phi_{i}}>G_{i(2 j)}\right) & \\
\leq & \int_{-\infty}^{\delta_{i 0}} p\left(\widetilde{\psi}_{i}\left(G_{i(2 j)}\right)<-x_{i}\right) \gamma_{i}\left(d x_{i}\right) \\
& +\int_{\delta_{i 0}}^{\infty} p\left(\psi_{i}\left(G_{i(2 j-1)}\right)>-x_{i}\right) \gamma_{i}\left(d x_{i}\right) .
\end{aligned}
$$

From Remark 1, we obtain

$$
\begin{aligned}
E_{\tau_{\phi}} & =\int_{0}^{\infty} p\left(\tau_{\phi}>t\right) d t \\
\leq & \sum_{j=0}^{\infty} \int_{G_{1 j}}^{G_{1(j+1)}} p\left(\tau_{\phi_{1}}>t\right) d t+\cdots \\
& +\sum_{j=0}^{\infty} \int_{G_{n j}}^{G_{n(j+1)}} p\left(\tau_{\phi_{n}}>t\right) d t
\end{aligned}
$$

where $G_{i 0}=0$; then

$$
\begin{aligned}
E_{\tau_{\phi}} \leq & \sum_{j=0}^{\infty} \int_{G_{1 j}}^{G_{1(j+1)}} p\left(\tau_{\phi_{1}}>G_{1 j}\right) d t+\cdots \\
& +\sum_{j=0}^{\infty} \int_{G_{n j}}^{G_{n(j+1)}} p\left(\tau_{\phi_{n}}>t\right) d t \\
= & \sum_{i=1}^{n} \sum_{j=0}^{\infty}\left(G_{i(j+1)}-G_{i j}\right) p\left(\tau_{\phi_{i}}>G_{i j}\right) \\
= & \sum_{i=1}^{n} \sum_{j=0}^{\infty}\left[\lambda_{i}\left(\vartheta_{i}^{j+1}-1\right)-\lambda_{i}\left(\vartheta_{i}^{j}-1\right)\right] p\left(\tau_{\phi_{i}}>G_{i j}\right) \\
= & \sum_{i=1}^{n} \sum_{j=0}^{\infty} \lambda_{i} \vartheta_{i}^{j}\left(\vartheta_{i}-1\right) p\left(\tau_{\phi_{i}}>G_{i j}\right) \\
= & \sum_{i=1}^{n}\left[\lambda_{i}\left(\vartheta_{i}-1\right) \sum_{j=0}^{\infty} \vartheta_{i}^{j} p\left(\tau_{\phi_{i}}>G_{i j}\right)\right] \\
= & \sum_{i=1}^{n}\left[\lambda_{i}\left(\vartheta_{i}-1\right)\right.
\end{aligned}
$$

$$
\begin{aligned}
\times & \left(p\left(\tau_{\phi_{i}}>D\right)+\vartheta_{i} p\left(\tau_{\phi_{i}}>G_{i 1}\right)\right. \\
& \left.\left.+\vartheta_{i}^{2} p\left(\tau_{\phi_{i}}>G_{i 2}\right)+\vartheta_{i}^{3} p\left(\tau_{\phi_{i}}>G_{i 3}\right)+\cdots\right)\right] .
\end{aligned}
$$

$$
\text { If } \delta_{i 0}<0 \text {, then we get }
$$$$
E_{\tau_{\phi}} \leq \sum_{i=1}^{n}\left[\lambda_{i}\left(\vartheta_{i}-1\right)\right.
$$$$
\times\left(p\left(\tau_{\phi_{i}}>0\right)+\vartheta_{i} p\left(\tau_{\phi_{i}}>G_{i 1}\right)\right.
$$$$
+\vartheta_{i}^{2}\left\{\int_{\delta_{i 0}}^{\infty} p\left(\psi_{i}\left(G_{i 1}\right)>-x_{i}\right) \gamma_{i}\left(d x_{i}\right)\right.
$$$$
\left.+\int_{-\infty}^{\delta_{i 0}} p\left(\widetilde{\psi}_{i}\left(G_{i 2}\right)<-x_{i}\right) \gamma_{i}\left(d x_{i}\right)\right\}
$$$$
+\vartheta_{i}^{3}\left\{\int_{\delta_{i 0}}^{\infty} p\left(\psi_{i}\left(G_{i 3}\right)>-x_{i}\right) \gamma_{i}\left(d x_{i}\right)\right.
$$$$
\left.+\int_{-\infty}^{\delta_{i 0}} p\left(\widetilde{\psi}_{i}\left(G_{i 2}\right)<-x_{i}\right) \gamma_{i}\left(d x_{i}\right)\right\}
$$$$
+\vartheta_{i}^{4}\left\{\int_{\delta_{i 0}}^{\infty} p\left(\psi_{i}\left(G_{i 3}\right)>-x_{i}\right) \gamma_{i}\left(d x_{i}\right)\right.
$$$$
\left.+\int_{-\infty}^{\delta_{i 0}} p\left(\widetilde{\psi}_{i}\left(G_{i 4}\right)<-x_{i}\right) \gamma_{i}\left(d x_{i}\right)\right\}
$$$$
+\cdots
$$$$
+\vartheta_{i}^{\mathscr{F}}\left\{\int_{\delta_{i 0}}^{\infty} p\left(\psi_{i}\left(G_{i(\mathscr{F}-1)}\right)>-x_{i}\right) \gamma_{i}\left(d x_{i}\right)\right.
$$$$
\left.+\int_{-\infty}^{\delta_{i 0}} p\left(\widetilde{\psi}_{i}\left(G_{i \mathscr{F}}\right)<-x_{i}\right) \gamma_{i}\left(d x_{i}\right)\right\}
$$$$
+\vartheta_{i}^{\mathscr{F}+1}\left\{\int_{\delta_{i 0}}^{\infty} p\left(\psi_{i}\left(G_{i(\mathscr{F}+1)}\right)>-x_{i}\right) \gamma_{i}\left(d x_{i}\right)\right.
$$$$
\left.+\int_{-\infty}^{\delta_{i 0}} p\left(\widetilde{\psi}_{i}\left(G_{i \mathscr{F}}\right)<-x_{i}\right) \gamma_{i}\left(d x_{i}\right)\right\}
$$$$
+\vartheta_{i}^{\mathscr{F}+2}\left\{\int_{\delta_{i 0}}^{\infty} p\left(\psi_{i}\left(G_{i(\mathscr{F}+1)}\right)>-x_{i}\right) \gamma_{i}\left(d x_{i}\right)\right.
$$$$
\left.+\int_{-\infty}^{\delta_{i 0}} p\left(\widetilde{\psi}_{i}\left(G_{i(\mathscr{F}+2)}\right)<-x_{i}\right) \gamma_{i}\left(d x_{i}\right)\right\}
$$$$
+\vartheta_{i}^{\mathscr{F}+3}\left\{\int_{\delta_{i 0}}^{\infty} p\left(\psi_{i}\left(G_{i(\mathscr{F}+3)}\right)>-x_{i}\right) \gamma_{i}\left(d x_{i}\right)\right.
$$$$
\left.+\int_{-\infty}^{\delta_{i 0}} p\left(\widetilde{\psi}_{i}\left(G_{i(\mathscr{F}+2)}\right)<-x_{i}\right) \gamma_{i}\left(d x_{i}\right)\right\}
$$

$+\cdots)]$ 
thus,

$$
\begin{aligned}
& E_{\tau_{\phi}} \leq \sum_{i=1}^{n}\left[\lambda_{i}\left(\vartheta_{i}-1\right)\right. \\
& \times\left(p\left(\tau_{\phi_{i}}>0\right)+\vartheta_{i} p\left(\tau_{\phi_{i}}>G_{i 1}\right)\right. \\
& +\vartheta_{i}^{2}\left(\vartheta_{i}+1\right) \\
& \times \int_{-\infty}^{\delta_{i 0}} p\left(\widetilde{\psi}_{i}\left(G_{i 2}\right)<-x_{i}\right) \gamma_{i}\left(d x_{i}\right)+\cdots \\
& +\mathcal{\vartheta}_{i}^{\mathscr{F}}\left(\mathcal{\vartheta}_{i}+1\right) \\
& \times \int_{-\infty}^{\delta_{i 0}} p\left(\widetilde{\psi}_{i}\left(G_{i \mathscr{F}}\right)<-x_{i}\right) \gamma_{i}\left(d x_{i}\right)+\cdots \\
& +\vartheta_{i}^{3}\left(\vartheta_{i}+1\right) \int_{\delta_{i 0}}^{\infty} p\left(\psi_{i}\left(G_{i 3}\right)>-x_{i}\right) \gamma_{i}\left(d x_{i}\right) \\
& +\vartheta_{i}^{5}\left(\vartheta_{i}+1\right) \\
& \times \int_{\delta_{i 0}}^{\infty} p\left(\psi_{i}\left(G_{i 5}\right)>-x_{i}\right) \gamma_{i}\left(d x_{i}\right)+\cdots \\
& +\vartheta_{i}^{\mathscr{F}+3}\left(\vartheta_{i}+1\right) \\
& \left.\left.\times \int_{\delta_{i 0}}^{\infty} p\left(\psi_{i}\left(G_{i(\mathscr{F}+3)}\right)>-x_{i}\right) \psi_{i}\left(d x_{i}\right)+\cdots\right)\right] .
\end{aligned}
$$

Leads to

$$
\begin{gathered}
E_{\tau_{\phi}} \leq \sum_{i=1}^{n}\left[\lambda_{i}\left(\vartheta_{i}-1\right)\right. \\
\times\left[{ }^{1} \widehat{g}_{i}+\left(\vartheta_{i}+1\right)\right. \\
\times\left(\int_{-\infty}^{\delta_{i 0}} \widehat{w}_{i}\left(x_{i}\right) \gamma_{i}\left(d x_{i}\right)\right. \\
+\int_{\delta_{i 0}}^{\infty} \widehat{q}_{i}\left(x_{i}\right) \gamma_{i}\left(d x_{i}\right) \\
\left.\left.\left.+\int_{\delta_{i 0}}^{\infty} \widehat{h}_{i}\left(x_{i}\right) \gamma_{i}\left(d x_{i}\right)\right)\right]\right] \\
=\sum_{i=1}^{n} \lambda_{i}\left(\vartheta_{i}-1\right)\left({ }^{1} \widehat{g}_{i}\right)
\end{gathered}
$$

$$
\begin{aligned}
+\sum_{i=1}^{n}\left[\lambda_{i}\left(\vartheta_{i}-1\right)\left(\vartheta_{i}+1\right)\right. \\
\times\left(\int_{-\infty}^{\delta_{i 0}} \widehat{w}_{i}\left(x_{i}\right) \gamma_{i}\left(d x_{i}\right)\right. \\
+\int_{\delta_{i 0}}^{\infty} \widehat{q}_{i}\left(x_{i}\right) \gamma_{i}\left(d x_{i}\right) \\
\left.\left.+\int_{\delta_{i 0}}^{\infty} \widehat{h}_{i}\left(x_{i}\right) \gamma_{i}\left(d x_{i}\right)\right)\right],
\end{aligned}
$$

where

$$
\begin{gathered}
{ }^{1} \widehat{g}_{i}=p\left(\tau_{\phi_{i}}>0\right)+\vartheta_{i} p\left(\tau_{\phi_{i}}>G_{i 1}\right) \\
+\vartheta_{i}^{2} \int_{\delta_{i 0}}^{\infty} p\left(\psi_{i}\left(G_{i 1}\right)>-x_{i}\right) \gamma_{i}\left(d x_{i}\right), \\
{ }^{1} \widehat{w}_{i}\left(x_{i}\right)=\sum_{j=1}^{\infty} \vartheta_{i}^{2 j} p\left(\widetilde{\psi}_{i}\left(G_{i(2 j)}\right)<-x_{i}\right), \\
{ }^{1} \widehat{q}_{i}\left(x_{i}\right)=\sum_{j=1}^{(\mathscr{F}-1) / 2} \vartheta_{i}^{2 j+1} p\left(\widetilde{\psi}_{i}\left(G_{i(2 j+1)}\right) \leq-x_{i}\right), \\
{ }^{1} \widehat{h}_{i}\left(x_{i}\right)=\sum_{j=(\mathscr{F}+1) / 2}^{\infty} \vartheta_{i}^{2 j+1} p\left(\psi_{i}\left(G_{i(2 j+1)}\right)>-x_{i}\right) .
\end{gathered}
$$

Then $E_{\tau_{\phi}}$ is finite if

$$
\begin{aligned}
\int_{\delta_{i 0}}^{\infty}[ & \sum_{j=(\mathscr{F}+1) / 2}^{\infty} \vartheta_{i}^{2 j+1} p\left(\psi_{i}\left(G_{i(2 j+1)}\right)>-x_{i}\right) \\
& \left.+\sum_{j=1}^{(\mathscr{F}-1) / 2} \vartheta_{i}^{2 j+1} p\left(\widetilde{\psi}_{i}\left(G_{i(2 j+1)}\right) \leq-x_{i}\right)\right] \gamma_{i}\left(d x_{i}\right),
\end{aligned}
$$

$\int_{-\infty}^{\delta_{i 0}} \sum_{j=1}^{\infty} \vartheta_{i}^{2 j} p\left(\widetilde{\psi}_{i}\left(G_{i(2 j)}\right)<-x_{i}\right) \gamma_{i}\left(d x_{i}\right)$, are finite. By similar way if $\delta_{i 0}>0$, then $E_{\tau_{\phi}}$ is finite if

$$
\begin{gathered}
E_{\tau_{\phi}} \leq \sum_{i=1}^{n}\left[\lambda_{i}\left(\vartheta_{i}-1\right)\left({ }^{2} \widehat{g}_{i}\right)+\lambda_{i}\left(\vartheta_{i}-1\right)\left(\vartheta_{i}+1\right)\right. \\
\times\left(\int_{-\infty}^{\delta^{i_{0}} 2} \widehat{w}_{i}\left(x_{i}\right) \gamma_{i}\left(d x_{i}\right)\right. \\
+\int_{-\infty}^{\delta_{i 0}} 2 \widehat{q}_{i}\left(x_{i}\right) \gamma_{i}\left(d x_{i}\right) \\
\left.\left.+\int_{\delta_{i 0}}^{\infty}{ }^{2} \widehat{h}_{i}\left(x_{i}\right) \gamma_{i}\left(d x_{i}\right)\right)\right]
\end{gathered}
$$


where

$$
\begin{array}{r}
{ }^{2} \widehat{g}_{i}=p\left(\tau_{\phi_{1}}>0\right)+\vartheta_{i} p\left(\tau_{\phi_{i}}>G_{i 1}\right) \\
+\vartheta_{i}^{2} \int_{\delta_{i 0}}^{\infty} p\left(\psi_{i}\left(G_{i 1}\right)>-x_{i}\right) \gamma_{i}\left(d x_{i}\right), \\
{ }^{2} \widehat{w}_{i}\left(x_{i}\right)=\sum_{j=1}^{\mathscr{F} / 2} \vartheta_{i}^{2 j} p\left(\psi_{i}\left(G_{i(2 j)}\right)<-x_{i}\right), \\
{ }^{2} \widehat{q}_{i}\left(x_{i}\right)=\sum_{j=\mathscr{F} / 2+1}^{\infty} \vartheta_{i}^{2 j} p\left(\widetilde{\psi}_{i}\left(G_{i(2 j)}\right) \leq-x_{i}\right), \\
{ }^{2} \widehat{h}_{i}\left(x_{i}\right)=\sum_{j=1}^{\infty} \vartheta_{i}^{2 j+1} p\left(\psi_{i}\left(G_{i(2 j+1)}\right)>-x_{i}\right) .
\end{array}
$$

And $E_{\tau_{\phi}}$ is finite if

$$
\begin{aligned}
\int_{-\infty}^{\delta_{i 0}}[ & \sum_{j=1}^{\mathscr{F} / 2} \vartheta_{i}^{2 j} p\left(\tilde{\psi}_{i}\left(G_{i(2 j)}\right)<-x_{i}\right) \\
& \left.+\sum_{j=\mathscr{F} / 2+1}^{\infty} \vartheta_{i}^{2 j} p\left(\tilde{\psi}_{i}\left(G_{i(2 j)}\right) \leq-x_{i}\right)\right] \gamma_{i}\left(d x_{i}\right),
\end{aligned}
$$

$\int_{\delta_{i 0}}^{\infty} \sum_{j=1}^{\infty} \vartheta_{i}^{2 j+1} p\left(\psi_{i}\left(G_{i(2 j+1)}\right)>-x_{i}\right)$, are finite.

Lemma 5 (see El-Rayes et al. [9]). If $a_{\widetilde{m}} \geq 0, a_{\widetilde{m}+1} \leq a_{\widetilde{m}}, \widetilde{m}=$ $1,2, \ldots$, and $\left\{\bar{d}_{\widetilde{m}}\right\}, \widetilde{m}=1,2, \ldots$, are a strictly increasing sequence of integer numbers with $\bar{d}_{0}=0$, then, for any $\widetilde{k}=$ $1,2, \ldots$,

$$
\begin{aligned}
\sum_{\widetilde{m}=\widetilde{k}}^{\infty}\left[\bar{d}_{\widetilde{m}+1}-\bar{d}_{\widetilde{m}}\right] a_{\bar{d}_{\tilde{m}+1}} & \leq \sum_{\widetilde{m}=\bar{d}_{\widetilde{k}}}^{\infty} a_{\widetilde{m}} \\
& \leq \sum_{\widetilde{m}=\widetilde{k}}^{\infty}\left[\bar{d}_{\widetilde{m}+1}-\bar{d}_{\widetilde{m}}\right] a_{\bar{d}_{\widetilde{m}}}
\end{aligned}
$$

where $\sum_{\widetilde{m}=\tilde{k}}^{\infty}\left[\bar{d}_{\widetilde{m}+1}-\bar{d}_{\widetilde{m}}\right] a_{\bar{d}_{\widetilde{m}+1}}, \sum_{\widetilde{m}=\bar{d}_{\tilde{k}}}^{\infty} a_{\widetilde{m}}$, and $\sum_{\widetilde{m}=\tilde{k}}^{\infty}\left[\bar{d}_{\widetilde{m}+1}-\right.$ $\left.\bar{d}_{\widetilde{m}}\right] a_{\bar{d}_{\bar{m}}}$ are vectors in formulas and they are taken to be row vectors. These vectors are defined as follows:

$$
\begin{aligned}
& \sum_{\widetilde{m}=\widetilde{k}}^{\infty}\left[\bar{d}_{\widetilde{m}+1}-\bar{d}_{\widetilde{m}}\right] a_{\bar{d}_{\widetilde{m}+1}} {\left[\sum_{\widetilde{m}=\widetilde{k}}^{\infty}\left[\bar{d}_{1(\widetilde{m}+1)}-\bar{d}_{1 \widetilde{m}}\right] a_{1 \bar{d}_{1(\widetilde{m}+1)},}\right.} \\
& \sum_{\widetilde{m}=\widetilde{k}}^{\infty}\left[\bar{d}_{2(\widetilde{m}+1)}-\bar{d}_{2 \widetilde{m}}\right] a_{2 \bar{d}_{2(\widetilde{m}+1)}, \ldots,}, \\
&\left.\sum_{\widetilde{m}=\widetilde{k}}^{\infty}\left[\bar{d}_{n(\widetilde{m}+1)}-\bar{d}_{n \widetilde{m}}\right] a_{n \bar{d}_{n(\widetilde{m}+1)}}\right],
\end{aligned}
$$

$$
\begin{aligned}
& \sum_{\widetilde{m}=\bar{d}_{\tilde{k}}}^{\infty} a_{\widetilde{m}}=\left[\sum_{\widetilde{m}=\bar{d}_{1 \tilde{k}}}^{\infty} a_{1 \widetilde{m}}, \sum_{\widetilde{m}=\bar{d}_{2 \widetilde{k}}}^{\infty} a_{2 \widetilde{m}}, \ldots, \sum_{\widetilde{m}=\bar{d}_{n \tilde{k}}}^{\infty} a_{n \widetilde{m}}\right], \\
& \sum_{\widetilde{m}=\widetilde{k}}^{\infty}\left[\bar{d}_{\widetilde{m}+1}-\bar{d}_{\widetilde{m}}\right] a_{\bar{d}_{\bar{m}}} \\
& =\left[\sum_{\widetilde{m}=\widetilde{k}}^{\infty}\left[\bar{d}_{1(\widetilde{m}+1)}-\bar{d}_{1 \widetilde{m}}\right] a_{1 \bar{d}_{1 \widetilde{m}}},\right. \\
& \sum_{\widetilde{m}=\widetilde{k}}^{\infty}\left[\bar{d}_{2(\widetilde{m}+1)}-\bar{d}_{2 \widetilde{m}}\right] a_{2 \bar{d}_{2 \widetilde{m}}}, \ldots
\end{aligned}
$$$$
\left.\sum_{\widetilde{m}=\widetilde{k}}^{\infty}\left[\bar{d}_{n(\widetilde{m}+1)}-\bar{d}_{n \widetilde{m}}\right] a_{n \bar{d}_{n \widetilde{m}}}\right] .
$$

Now, we will discuss under what conditions of the chosen search plan should be satisfied to make the previous integrals in Theorem 4 are indeed finite. This is a crucial issue related to the existence of a finite search plan.

Theorem 6. The chosen search plan should satisfy ${ }^{2} \widehat{h}(x) \leq$ $L(|x|),{ }^{2} \widehat{q}(x) \leq \widetilde{L}(|x|)$, if $\delta_{i 0}>0$ and ${ }^{1} \widehat{w}(x) \leq M(|x|),{ }^{1} \widehat{h}(x)$ $\leq \widetilde{M}(|x|)$ if $\delta_{i 0}<0$, where $L(|x|), \widetilde{L}(|x|), M(|x|)$, and $\widetilde{M}(|x|)$ are vectors of linear functions given by ${ }^{1} \widehat{w}(x)={ }^{1} \widehat{w}_{1}$ $\left.\left(x_{1}\right),{ }^{1} \widehat{w}_{2}\left(x_{2}\right), \ldots,{ }^{1} \widehat{w}_{n}\left(x_{n}\right)\right],{ }^{1} \widehat{h}(x)=\left[{ }^{1} \widehat{h}_{1}\left(x_{1}\right),{ }^{1} \widehat{h}_{2}\left(x_{2}\right), \ldots\right.$, $\left.{ }^{1} \widehat{h}_{n}\left(x_{n}\right)\right],{ }^{2} \widehat{h}(x)=\left[{ }^{2} \widehat{h}_{1}\left(x_{1}\right),{ }^{2} \widehat{h}_{2}\left(x_{2}\right), \ldots,{ }^{2} \widehat{h}_{n}\left(x_{n}\right)\right],{ }^{2} \widehat{q}(x)=$ $\left[{ }^{2} \widehat{q}_{1}\left(x_{1}\right),{ }^{2} \widehat{q}_{2}\left(x_{2}\right), \ldots,{ }^{2} \widehat{q}_{n}\left(x_{n}\right)\right], L(|x|)=\left[L_{1}\left(\left|x_{1}\right|\right), L_{2}\left(\left|x_{2}\right|\right)\right.$, $\left.\ldots, L_{n}\left(\left|x_{n}\right|\right)\right], \widetilde{L}(|x|)=\left[\widetilde{L}_{1}\left(\left|x_{1}\right|\right), \widetilde{L}_{2}\left(\left|x_{2}\right|\right), \ldots, \widetilde{L}_{n}\left(\left|x_{n}\right|\right)\right], M$ $(|x|)=\left[M_{1}\left(\left|x_{1}\right|\right), M_{2}\left(\left|x_{2}\right|\right), \ldots, M_{n}\left(\left|x_{n}\right|\right)\right]$ and $\widetilde{M}(|x|)=$ $\left[\widetilde{M}_{1}\left(\left|x_{1}\right|\right), \widetilde{M}_{2}\left(\left|x_{2}\right|\right), \ldots, \widetilde{M}_{n}\left(\left|x_{n}\right|\right)\right]$.

Proof. We will prove this theorem for ${ }^{2} \widehat{h}(x)$ when $\delta_{i 0}>0$, where ${ }^{2} \widehat{h}(x)=\left[{ }^{2} \widehat{h}_{1}\left(x_{1}\right),{ }^{2} \widehat{h}_{2}\left(x_{2}\right), \ldots,{ }^{2} \widehat{h}_{n}\left(x_{n}\right)\right]=\left[\sum_{j=1}^{\infty} \vartheta_{1}^{2 j+1} p\right.$ $\left(\psi_{1}\left(G_{1(2 j+1)}\right)>-x_{1}\right), \sum_{j=1}^{\infty} \vartheta_{2}^{2 j+1} p\left(\psi_{2}\left(G_{2(2 j+1)}\right)>-x_{2}\right), \ldots$, $\left.\sum_{j=1}^{\infty} \vartheta_{n}^{2 j+1} p\left(\psi_{n}\left(G_{n(2 j+1)}\right)>-x_{n}\right)\right]$, and we obtain the following cases.

(I) If $x_{i}>\delta_{i 0}$, then we have $\left[{ }^{2} \widehat{h}_{1}\left(x_{1}\right),{ }^{2} \widehat{h}_{2}\left(x_{2}\right)\right.$, $\left.\ldots,{ }^{2} \widehat{h}_{n}\left(x_{n}\right)\right]=\left[{ }^{2} \widehat{h}_{1}\left(\delta_{10}\right),{ }^{2} \widehat{h}_{2}\left(\delta_{20}\right), \ldots,{ }^{2} \widehat{h}_{n}\left(\delta_{n 0}\right)\right]+\left[\sum_{j=1}^{\infty} \vartheta_{1}^{2 j+1}\right.$ $p\left(-x_{1}<\psi_{1}\left(G_{1(2 j+1)}\right) \leq-\delta_{10}\right), \sum_{j=1}^{\infty} \vartheta_{2}^{2 j+1} p\left(-x_{2}<\psi_{2}\right.$ $\left.\left(G_{2(2 j+1)}\right) \leq-\delta_{20}\right), \ldots, \sum_{j=1}^{\infty} \vartheta_{n}^{2 j+1} p\left(-x_{n}<\psi_{n}\left(G_{n(2 j+1)}\right) \leq\right.$ $\left.\left.-\delta_{n 0}\right)\right]$.

(II) If $0 \leq x_{i} \leq \delta_{i 0}$, then we have [ ${ }^{2} \widehat{h}_{1}\left(x_{1}\right),{ }^{2} \widehat{h}_{2}\left(x_{2}\right)$, $\left.\ldots,{ }^{2} \widehat{h}_{n}\left(x_{n}\right)\right]=\left[{ }^{2} \widehat{h}_{1}(0),{ }^{2} \widehat{h}_{2}(0), \ldots,{ }^{2} \widehat{h}_{n}(0)\right]+\left[\sum_{j=1}^{\infty} \vartheta_{1}^{2 j+1}\right.$ $p\left(-x_{1}<\psi_{1}\left(G_{1(2 j+1)}\right) \leq 0\right), \sum_{j=1}^{\infty} \vartheta_{2}^{2 j+1} p\left(-x_{2}<\psi_{2}\left(G_{2(2 j+1)}\right) \leq\right.$ $\left.0), \ldots, \sum_{j=1}^{\infty} \vartheta_{n}^{2 j+1} p\left(-x_{n}<\psi_{n}\left(G_{n(2 j+1)}\right) \leq 0\right)\right]$. 
(III) If $x_{i} \leq \delta_{i 0}$, then we get $\left[{ }^{2} \widehat{h}_{1}\left(x_{1}\right),{ }^{2} \widehat{h}_{2}\left(x_{2}\right)\right.$, $\left.\ldots,{ }^{2} \widehat{h}_{n}\left(x_{n}\right)\right]=\left[{ }^{2} \widehat{h}_{1}(0),{ }^{2} \widehat{h}_{2}(0), \ldots,{ }^{2} \widehat{h}_{n}(0)\right]-\left[\sum_{j=1}^{\infty} \vartheta_{1}^{2 j+1} p(0<\right.$ $\left.\psi_{1}\left(G_{1(2 j+1)}\right) \leq-x_{1}\right), \sum_{j=1}^{\infty} \vartheta_{2}^{2 j+1} p\left(0<\psi_{2}\left(G_{2(2 j+1)}\right) \leq-x_{2}\right)$, $\left.\ldots, \sum_{j=1}^{\infty} \vartheta_{n}^{2 j+1} p\left(0<\psi_{n}\left(G_{n(2 j+1)}\right) \leq-x_{n}\right)\right]$.

From (II) we have $\left[{ }^{2} \widehat{h}_{1}\left(x_{1}\right),{ }^{2} \widehat{h}_{2}\left(x_{2}\right), \ldots,{ }^{2} \widehat{h}_{n}\left(x_{n}\right)\right] \leq\left[{ }^{2} \widehat{h}_{1}\right.$ $\left.(0),{ }^{2} \widehat{h}_{2}(0), \ldots,{ }^{2} \widehat{h}_{n}(0)\right]$, and for $x_{i} \geq \delta_{i 0}\left[{ }^{2} \widehat{h}_{1}\left(x_{1}\right),{ }^{2} \widehat{h}_{2}\right.$ $\left.\left(x_{2}\right), \ldots,{ }^{2} \widehat{h}_{n}\left(x_{n}\right)\right]=\left[{ }^{2} \widehat{h}_{1}\left(\delta_{10}\right),{ }^{2} \widehat{h}_{2}\left(\delta_{20}\right), \ldots,{ }^{2} \widehat{h}_{n}\left(\delta_{n 0}\right)\right]+$ $\left[\sum_{j=1}^{\infty} \vartheta_{1}^{2 j+1} p\left(-x_{1}<\psi_{1}\left(G_{1(2 j+1)}\right) \leq-\delta_{10}\right), \sum_{j=1}^{\infty} \vartheta_{2}^{2 j+1} p\left(-x_{2}<\right.\right.$ $\left.\psi_{2}\left(G_{2(2 j+1)}\right) \leq-\delta_{20}\right), \ldots, \sum_{j=1}^{\infty} \vartheta_{n}^{2 j+1} p\left(-x_{n}<\psi_{n}\left(G_{n(2 j+1)}\right) \leq\right.$ $\left.\left.-\delta_{n 0}\right)\right]$, but $\left[{ }^{2} \widehat{h}_{1}\left(x_{1}\right),{ }^{2} \widehat{h}_{2}\left(x_{2}\right), \ldots,{ }^{2} \widehat{h}_{n}\left(x_{n}\right)\right]=\left[{ }^{2} \widehat{h}_{1}(0),{ }^{2} \widehat{h}_{2}(0)\right.$, $\left.\ldots,{ }^{2} \widehat{h}_{n}(0)\right]+\left[\sum_{j=1}^{\infty} \vartheta_{1}^{2 j+1} p\left(-x_{1}<\psi_{1}\left(G_{1(2 j+1)}\right) \leq 0\right), \sum_{j=1}^{\infty}\right.$ $\vartheta_{2}^{2 j+1} p\left(-x_{2}<\psi_{2}\left(G_{2(2 j+1)}\right) \leq 0\right), \ldots, \sum_{j=1}^{\infty} \vartheta_{n}^{2 j+1} p\left(-x_{n}<\psi_{n}\right.$ $\left.\left.\left(G_{n(2 j+1)}\right) \leq 0\right)\right]$. Consequently, from Theorem 3, we get $\left[{ }^{2} \widehat{h}_{1}(0),{ }^{2} \widehat{h}_{2}(0), \ldots,{ }^{2} \widehat{h}_{n}(0)\right]=\left[\sum_{j=1}^{\infty} \vartheta_{1}^{2 j+1} p\left(\psi_{1}\left(G_{1(2 j+1)}\right)>\right.\right.$ $0), \sum_{j=1}^{\infty} \vartheta_{2}^{2 j+1} p\left(\psi_{2}\left(G_{2(2 j+1)}\right) \quad>0\right), \ldots, \sum_{j=1}^{\infty} \vartheta_{n}^{2 j+1} p\left(\psi_{n}\right.$ $\left.\left.\left(G_{n(2 j+1)}\right)>0\right)\right] \leq\left[\sum_{j=1}^{\infty} \vartheta_{1}^{2 j+1} \varepsilon_{1}^{G_{1(2 j+1)}}, \sum_{j=1}^{\infty} \vartheta_{2}^{2 j+1} \varepsilon_{2}^{G_{2(2 j+1)}}\right.$, $\left.\ldots, \sum_{j=1}^{\infty} \vartheta_{n}^{2 j+1} \varepsilon_{n}^{G_{n(2 j+1)}}\right] \leq\left[\vartheta_{1}^{3} /\left(\vartheta_{1}^{2}-1\right), \vartheta_{2}^{3} /\left(\vartheta_{2}^{2}-1\right), \ldots, \vartheta_{n}^{3} /\left(\vartheta_{n}^{2}-\right.\right.$ 1)], $0<\varepsilon_{i}<1$.

Suppose the following holds:

(i) $\bar{d}_{i \xi_{i}}=G_{i\left(2 \xi_{i}+1\right)} / \sigma_{i}=\mu_{i}\left(\vartheta_{i}^{2 \xi_{i}+1}-1\right)$,

(ii) $\mu_{i}\left(\xi_{i}\right)=\psi_{i}\left(\xi_{i} \sigma_{i}\right) / 2=\sum_{j=1}^{\xi_{i}} X_{i j}$, where $\left\{X_{i j}\right\}, j=$ $1,2, \ldots$, is a sequence of independent identically distributed random variables,

(iii) $a_{i}\left(\xi_{i}\right)=p\left(-x_{i} / 2<\mu_{i}\left(\xi_{i}\right) \leq 0\right)=\sum_{\mathscr{F}=0}^{\left|x_{i}\right| / 2} p(-(\mathscr{F}+1)<$ $\left.\mu_{i}\left(\xi_{i}\right) \leq-\mathscr{F}\right)$

(iv) $\widetilde{m}$ is an integer such that $\bar{d}_{i \widetilde{m}}=r_{i 1}\left|x_{i}\right|+r_{i 2}$,

(v) $\widetilde{\alpha}_{i}=\vartheta_{i}^{2} / \mu_{i}\left(\vartheta_{i}-1\right)$,

(vi) $U_{i}(\mathscr{F}, \mathscr{F}+1)=\sum_{\xi_{i}=0}^{\infty} p\left(-(\mathscr{F}+1)<\mu_{i}\left(\xi_{i}\right) \leq-\mathscr{F}\right)$.

Thus, from Theorem 2, we have $a_{i}\left(\xi_{i}\right)$ is nonincreasing if $\xi_{i}>\bar{d}_{i \xi_{i}}$. By applying Lemma 5, we have [ ${ }^{2} \widehat{h}_{1}\left(x_{1}\right),{ }^{2} \widehat{h}_{2}\left(x_{2}\right)$, $\left.\ldots,{ }^{2} \widehat{h}_{n}\left(x_{n}\right)\right]-\left[{ }^{2} \widehat{h}_{1}(0),{ }^{2} \widehat{h}_{2}(0), \ldots,{ }^{2} \widehat{h}_{n}(0)\right]=\left[\sum_{j=1}^{\infty} \vartheta_{1}^{2 j+1} p\right.$ $\left(-x_{1}<\psi_{1}\left(G_{1(2 j+1)}\right) \leq 0\right), \sum_{j=1}^{\infty} \vartheta_{2}^{2 j+1} p\left(-x_{2}<\psi_{2}\left(G_{2(2 j+1)}\right)\right.$ $\left.\leq 0), \ldots, \sum_{j=1}^{\infty} \vartheta_{n}^{2 j+1} p\left(-x_{n}<\psi_{n}\left(G_{n(2 j+1)}\right) \leq 0\right)\right]=\left[\sum_{\xi_{1}=1}^{\widetilde{m}}\right.$ $\left.\vartheta_{1}^{22 \xi_{1}+1} a_{1}\left(\bar{d}_{1 \xi_{1}}\right), \sum_{\xi_{2}=1}^{\widetilde{m}} \vartheta_{2}^{2 \xi_{2}+1} a_{2}\left(\bar{d}_{2 \xi_{2}}\right), \ldots, \sum_{\xi_{n}=1}^{\widetilde{m}} \vartheta_{n}^{2 \xi_{n}+1} a_{n}\left(\bar{d}_{n \xi_{n}}\right)\right]$ $+\left[\sum_{\xi_{1}=\widetilde{m}+1}^{\infty} \vartheta_{1}^{2 \xi_{1}+1} a_{1}\left(\bar{d}_{1 \xi_{1}}\right), \sum_{\xi_{2}=\widetilde{m}+1}^{\infty} \vartheta_{2}^{2 \xi_{2}+1} a_{2}\left(\bar{d}_{2 \xi_{2}}\right), \ldots, \sum_{\xi_{n}=\widetilde{m}+1}^{\infty}\right.$ $\left.\vartheta_{n}^{2 \xi_{n}+1} a_{n}\left(\bar{d}_{n \xi_{n}}\right)\right] \leq\left[\sum_{\xi_{1}=1}^{\widetilde{m}} \vartheta_{1}^{2 \xi_{1}+1}, \sum_{\xi_{2}=1}^{\widetilde{m}} \vartheta_{2}^{2 \xi_{2}+1}, \ldots, \sum_{\xi_{n}=1}^{\widetilde{m}} \vartheta_{n}^{2 \xi_{n}+1}\right]$ $+\left[\widetilde{\alpha}_{1} \sum_{\xi_{1}=\widetilde{m}+1}^{\infty}\left(\bar{d}_{1 \xi_{1}}-\bar{d}_{1\left(\xi_{1}-1\right)}\right) a_{1}\left(\bar{d}_{1 \xi_{1}}\right), \widetilde{\alpha}_{2} \sum_{\xi_{2}=\widetilde{m}+1}^{\infty}\left(\bar{d}_{2 \xi_{2}}-\right.\right.$ $\left.\left.\bar{d}_{2\left(\xi_{2}-1\right)}\right) a_{2}\left(\bar{d}_{2 \xi_{2}}\right), \ldots, \widetilde{\alpha}_{n} \sum_{\xi_{n}=\widetilde{m}+1}^{\infty}\left(\bar{d}_{n \xi_{n}}-\bar{d}_{n\left(\xi_{n}-1\right)}\right) a_{n}\left(\bar{d}_{n \xi_{n}}\right)\right]$ $\leq\left[\sum_{\xi_{1}=1}^{\widetilde{m}} \vartheta_{1}^{2 \xi_{1}+1}, \sum_{\xi_{2}=1}^{\widetilde{m}} \vartheta_{2}^{2 \xi_{2}+1}, \ldots, \sum_{\xi_{n}=1}^{\widetilde{m}} \vartheta_{n}^{2 \xi_{n}+1}\right]+\left[\widetilde{\alpha}_{1} \sum_{\xi_{1}}^{\infty} \bar{d}_{1 \bar{m}}\right.$ $\left.a_{1}\left(\xi_{1}\right), \widetilde{\alpha}_{2} \sum_{\xi_{2}=\bar{d}_{2 \widetilde{m}}}^{\infty} a_{2}\left(\xi_{2}\right), \ldots, \widetilde{\alpha}_{n} \sum_{\xi_{n}=\bar{d}_{n \tilde{m}}}^{\infty} a_{n}\left(\xi_{n}\right)\right] \leq\left[\sum_{\xi_{1}=1}^{\bar{m}}\right.$ $\left.\vartheta_{1}^{2 \xi_{1}+1}, \sum_{\xi_{2}=1}^{\widetilde{m}} \vartheta_{2}^{2 \xi_{2}+1}, \ldots, \sum_{\xi_{n}=1}^{\widetilde{m}} \vartheta_{n}^{2 \xi_{n}+1}\right]+\left[\widetilde{\alpha}_{1} \sum_{\mathscr{F}_{1}=0}^{\left|x_{1}\right| / 2} U_{1}(\mathscr{F}, \mathscr{F}+\right.$ 1), $\left.\widetilde{\alpha}_{2} \sum_{\mathscr{F}=0}^{\left|x_{2}\right| / 2} U_{2}(\mathscr{F}, \mathscr{F}+1), \ldots, \widetilde{\alpha}_{n} \sum_{\mathscr{F}=0}^{\left|x_{n}\right| / 2} U_{n}(\mathscr{F}, \mathscr{F}+1)\right]$.

Since $U_{i}(\mathscr{F}, \mathscr{F}+1)$ satisfies the conditions of renewal theorem as in Feller [28], then $U_{i}(\mathscr{F}, \mathscr{F}+1)$ is bounded for all $\mathscr{F}, i=1,2, \ldots, n$, by a constant so ${ }^{2} \widehat{h}(x)=$ $\left[q_{1}\left(x_{1}\right), q_{2}\left(x_{2}\right), \ldots, q_{n}\left(x_{n}\right)\right] \leq\left[{ }^{2} \widehat{h}_{1}\left(\delta_{10}\right),{ }^{2} \widehat{h}_{2}\left(\delta_{20}\right), \ldots,{ }^{2} \widehat{h}_{n}\right.$ $\left.\left(\delta_{n 0}\right)\right]+\left[{ }^{2} \Lambda_{11},{ }^{2} \Lambda_{21}, \ldots,{ }^{2} \Lambda_{n 1}\right]+\left[{ }^{2} \Lambda_{12}\left|x_{1}\right|,{ }^{2} \Lambda_{22}\left|x_{2}\right|\right.$, $\left.\ldots,{ }^{2} \Lambda_{n 2}\left|x_{n}\right|\right]=\left[L_{1}\left(\left|x_{1}\right|\right), L_{2}\left(\left|x_{2}\right|\right), \ldots, L_{n}\left(\left|x_{n}\right|\right)\right]=L(|x|)$, then, ${ }^{2} \widehat{h}(x) \leq L(|x|)$. Similar to the previous analysis way we can prove that ${ }^{2} \widehat{q}(x) \leq \widetilde{L}(|x|)$ and also ${ }^{1} \widehat{w}(x) \leq M(|x|)$, ${ }^{1} \widehat{h}(x) \leq \widetilde{M}(|x|)$ if $\delta_{i 0}<0$. Therefore, the proof is completed.

Theorem 7. If there exists a finite search plan $\phi(t)=$ $\left(\phi_{1}(t), \phi_{2}(t), \ldots, \phi_{n}(t)\right) \in \Phi(t)$, then $E\left(\left|X_{0}\right|\right)$ is finite.

Proof. For $E_{\tau_{\phi_{i}}}<\infty, i=1,2, \ldots, n$, we have $p\left(\tau_{\phi}\right.$ is finite $)=$ 1 and so $p$ (one of $\tau_{\phi_{i}}$ is finite, $i=1,2, \ldots, n$ ) $=1$. Therefore,

$$
\begin{aligned}
p\left(\bigcup_{i=1}^{n}\left(\tau_{\phi_{i}}<\infty\right)\right)= & \sum_{i=1}^{n} p\left(\tau_{\phi_{i}}<\infty\right) \\
& -\sum_{i, \bar{\beta}} p\left(\left(\tau_{\phi_{i}}<\infty\right) \cap\left(\tau_{\phi_{\bar{\beta}}}<\infty\right)\right)+\cdots \\
& +(-1)^{n} p\left(\bigcap_{i=1}^{n}\left(\tau_{\phi_{i}}<\infty\right)\right) .
\end{aligned}
$$

Since $\tau_{\phi_{i}}<\infty, i=1,2, \ldots, n$, are mutually exclusive events, then

$$
\sum_{i=1}^{n} p\left(\tau_{\phi_{i}}<\infty\right)=1
$$

Consequently, $p\left(\tau_{\phi_{i}}\right.$ is finite $)=1$ or $p\left(\tau_{\phi_{\theta}}\right.$ is finite $)=$ 1 for all $i \neq \theta, i, \theta=1,2, \ldots, n$. If $p\left(\tau_{\phi_{i}}\right.$ is finite $)=1$ then $X_{0}=\phi_{i}\left(\tau_{\phi_{i}}\right)-W\left(\tau_{\phi_{i}}\right)$ with probability one and $\left|X_{0}\right| \leq$ $\left|\phi_{i}\left(\tau_{\phi_{i}}\right)\right|+\left|W\left(\tau_{\phi_{i}}\right)\right| \leq \tau_{\phi_{i}}+\left|W\left(\tau_{\phi_{i}}\right)\right|$ that leads to $E\left(\left|X_{0}\right|\right) \leq$ $E_{\tau_{\phi_{i}}}+E\left(\left|W\left(\tau_{\phi_{i}}\right)\right|\right)$. But $W\left(\tau_{\phi_{i}}\right)<\tau_{\phi_{i}}$, then $E\left(\left|W\left(\tau_{\phi_{i}}\right)\right|\right)<E_{\tau_{\phi_{i}}}$, and $E\left(\left|X_{0}\right|\right)$ is finite.

On the other hand, if $p\left(\tau_{\phi_{\theta}}\right.$ is finite $)=1$ then $X_{0}=$ $\phi_{\theta}\left(\tau_{\phi_{\theta}}\right)-W\left(\tau_{\phi_{\theta}}\right)$ with probability one, and by the same manner we can get $E\left(\left|X_{0}\right|\right)$ is finite.

Remark 8. A direct consequence of Theorems 4, 6, and 7 satisfies the existence of a finite search plan if and only if $E\left(\left|X_{0}\right|\right)$ is finite.

\section{Existence of an Optimal Search Plan}

The goal of the searching strategy could minimize the expected value of the first meeting time between one of the searchers and the target. Therefore, the main problem here is to find a search paths $\phi_{i}(t), i=1,2, \ldots, m$. If such a search paths exists, we call it optimal search paths.

Definition 9. Let $\left\{\phi_{i h}(t)\right\}_{h \geq 1} \in \Phi_{1}(t), i=1,2, \ldots, m$, be sequences of search plans; we say that $\phi_{i h}(t)$ converges to $\phi_{i}(t)$ as $h$ tends to $\infty$ if and only if, for any $t \in I^{+}, \phi_{i h}(t)$ converges to $\phi_{i}(t)$ uniformly on every compact space (El-Rayes et al. [9]). 
Theorem 10. Let for any $t \in I^{+}$and $W(t)$ be a one-dimensional random walk. Then, the mapping,

$$
\left(\phi_{1}(t), \phi_{2}(t), \ldots, \phi_{n}(t)\right) \longrightarrow E_{\tau_{\phi}} \in I^{+}
$$

is lower semicontinuous on $\Phi_{1}(t)$.

Proof. Let $B\left(\phi_{i}, t\right)$ be the indicator function of the set $\left\{\tau_{\phi_{i}}>\right.$ $t, i=1,2, \ldots, n\}$; by the Fatou-Lesbesque theorem, we get

$$
\begin{aligned}
E_{\tau_{\phi}} & =E\left[\sum_{t=0}^{\infty} B\left(\phi_{i}, t\right)\right] \\
& =E\left[\sum_{t=0}^{\infty} \lim \inf B\left(\phi_{i h}(t), t\right)\right] \\
& \leq \sum_{t=0}^{\infty} \lim _{h \rightarrow \infty} \inf E\left(1_{\left\{\tau_{\phi_{h}}>t\right\}}\right),
\end{aligned}
$$

for any sequences $\phi_{i h} \rightarrow \phi_{i}$ in $\Phi_{1}(t)$, where $\Phi_{1}(t)$ is sequentially compact; see El-Rayes et al. [9]. Thus the mapping $\left(\phi_{1}(t), \phi_{2}(t), \ldots, \phi_{n}(t)\right) \rightarrow E_{\tau_{\phi}}$ is lower semicontinuous mapping on $\Phi_{1}(t)$ and this completed the proof.

\section{Optimal Search Plan}

In this section, we will find the optimal search plan that minimize the expected value of the first meeting time.

Since $\tau_{\phi}$ depends on infimum either one of the functions $\phi_{i}(t), i=1,2, \ldots, n$, then we need to minimize $E_{\tau_{\phi}}$. That happens when we obtain the optimal search plan $\phi^{*}(t)=$ $\left\{\phi_{1}^{*}(t), \phi_{2}^{*}(t), \ldots, \phi_{n}^{*}(t)\right\}$, where $\phi_{i}(t)$ is a function on a distance $H_{i j}, i=1,2, \ldots, n, 1 \leq j \leq \mathscr{F} / 2$. Therefore, it can be seen that the vector $\left(\phi_{1}(t), \phi_{2}(t), \ldots, \phi_{n}(t)\right)$ are $n$-distinct objective functions. Since the speed of the $n$-searchers (search team) is $v_{i}=1, i=1,2, \ldots, n$, then the objective functions $\phi_{i}(t), i=1,2, \ldots, n$, are distinct set of constrains given from Cases 1 and 2 . In this problem, we consider the constraint $G_{i(2 j-1)} \leq t \leq G_{i(2 j)}, i=1,2, \ldots, n, 1 \leq j \leq \mathscr{F} / 2$. Then, the problem takes the form

$$
\begin{array}{r}
\min \left(\phi_{1}(t), \phi_{2}(t), \ldots, \phi_{n}(t)\right) \\
\text { subject to } t \in T=\left\{t \in I^{+} \mid G_{i(2 j-1)} \leq t \leq G_{i(2 j)},\right. \\
\left.\quad i=1,2, \ldots, n, 1 \leq j \leq \frac{\mathscr{F}}{2}\right\},
\end{array}
$$

where $t$ is an $n$-dimensional vector of the decision variables, $\phi_{1}(t), \phi_{2}(t), \ldots, \phi_{n}(t)$ are $n$-distinct objective functions of the decision vector of inequality constraints, and $T$ is the feasible set of constrained decisions.

But $v_{i}=1, i=1,2, \ldots, n$, that leads to $t=H_{i j}$, $i=1,2, \ldots, n, 1 \leq j \leq \mathscr{F} / 2$; then the above problem is represented as the following multiobjective nonlinear programming problem (MONLP):

\section{MONLP:}

$$
\begin{aligned}
& \min \left(\Xi_{1}\left(H_{1 j}\right), \Xi_{2}\left(H_{2 j}\right), \ldots, \Xi_{n}\left(H_{n j}\right)\right), \\
& \text { subject to } \quad G_{i(2 j-1)} \leq H_{i j} \leq G_{i(2 j)}, \\
& \qquad H_{i j} \in I^{+}, \quad i=1,2, \ldots, n, 1 \leq j \leq \frac{\mathscr{F}}{2}, \\
& \text { where } \quad \Xi_{i}\left(H_{i j}\right)=H_{i(2 j+1)}-\delta_{i 0}-\left[t-G_{i(2 j-1)}\right] .
\end{aligned}
$$

However, on formulating the MONLP which closely describes and represents the real decision situation, various factors of the real system should be reflected in the description of the objective functions and the constraints. Naturally, these objective functions and the constraints involve many variables and parameters whose possible values may be assigned by the experts. In the conventional approach, such parameters are changed according to the variables in an experimental and/or subjective manner through the experts understanding the nature of the variables.

Substituting $G_{i j}=b \omega_{i}\left(\vartheta_{i}^{j}-1\right)$ in the above MONLP we have

\section{MONLP(I):}

$$
\begin{array}{r}
\min \left(\Xi_{1}\left(\vartheta_{1} ; \omega_{1}\right), \Xi_{2}\left(\vartheta_{2} ; \omega_{2}\right), \ldots, \Xi_{n}\left(\vartheta_{n} ; \omega_{n}\right)\right), \\
\text { subject to } b \omega_{i}\left(\vartheta_{i}^{2 j-1}-1\right) \leq H_{i j}, \quad H_{i j} \leq b \omega_{i}\left(\vartheta_{i}^{2 j}-1\right), \\
H_{i j} \in I^{+}, \quad i=1,2, \ldots, n, \quad 1 \leq j \leq \frac{\mathscr{F}}{2},
\end{array}
$$

where $\Xi_{i}\left(H_{i j}\right)=H_{i(2 j+1)}-\delta_{i 0}$

$$
-\left[t-b \omega_{i}\left(\vartheta_{i}^{2 j-1}-1\right)\right]
$$

where the variable $\vartheta_{i}$ takes +ve numbers greater than 1 and the parameter $\omega_{i}$ is the least positive integer such that $\omega_{i}(1-$ $\left.\left(\vartheta_{i}-1\right) /\left(\vartheta_{i}+1\right)\right) / 2$ is positive number; $H_{i j} \geq 0$ for all $i=$ $1,2, \ldots, n, 1 \leq j \leq \mathscr{F} / 2$. Furthermore, $H_{i j}$ is a function of the variable $\vartheta_{i}$ and the parameter $\omega_{i}$, where

$$
\begin{aligned}
H_{i j}= & (-1)^{j+1} C_{i}\left[G_{i j}+1+(-1)^{j+1}\right]+\delta_{i 0} \\
= & (-1)^{j+1}\left(\frac{\vartheta_{i}-1}{\vartheta_{i}+1}\right)\left[b \omega_{i}\left(\theta_{i}^{j}-1\right)+1+(-1)^{j+1}\right] \\
& +\delta_{i 0} .
\end{aligned}
$$


Then, the previous MONLP(I) takes the form

MONLP(II):

$\min \left(\Xi_{1}\left(\vartheta_{1} ; \varpi_{1}\right), \Xi_{2}\left(\vartheta_{2} ; \varpi_{2}\right), \ldots, \Xi_{n}\left(\vartheta_{n} ; \varpi_{n}\right)\right)$,

subject to $b \omega_{i}\left(\vartheta_{i}^{2 j-1}-1\right)$

$$
\begin{aligned}
& \leq\left(h_{1}-1\right)\left(\frac{\vartheta_{i}-1}{\vartheta_{i}+1}\right) \\
& \times\left[b \omega_{i}\left(9_{i}^{j}-1\right)+h_{1}\right]+\delta_{i 0}, \\
& b{\omega_{i}}_{i}\left(\vartheta_{i}^{2 j}-1\right) \geq\left(h_{1}-1\right)\left(\frac{\vartheta_{i}-1}{\vartheta_{i}+1}\right) \\
& \times\left[b \omega_{i}\left(\vartheta_{i}^{j}-1\right)+h_{1}\right] \\
& +\delta_{i 0},
\end{aligned}
$$

where $\Xi_{i}\left(\vartheta_{i} ; ळ_{i}\right)=\left(h_{3}\right)\left(\frac{\vartheta_{i}-1}{\vartheta_{i}+1}\right)$

$$
\begin{aligned}
& \times\left[b{\omega_{i}}_{i}\left(\vartheta_{i}^{2 j-1}-1\right)+h_{4}\right] \\
& -2 \delta_{i 0}+\left(h_{2}\right)\left(\frac{\vartheta_{i}-1}{\vartheta_{i}+1}\right) \\
& \times\left[b \omega_{i}\left(\vartheta_{i}^{j}-1\right)+h_{5}\right] \\
& +b{\omega_{i}}_{i}\left(\vartheta_{i}^{2 j-1}-1\right),
\end{aligned}
$$$$
h_{1}=1+(-1)^{j+1}, \quad h_{2}=(-1)^{j+2}, \quad h_{3}=(-1)^{2 j+1},
$$$$
h_{4}=1+(-1)^{2 j}, \quad h_{5}=1+(-1)^{j}, \quad \vartheta_{i}>1,
$$$$
\varrho_{i}>0 \quad \forall i=1,2, \ldots, n, \quad b=1,2, \ldots, \quad 1 \leq j \leq \frac{\mathscr{F}}{2} .
$$

Choosing a certain weights $\mho_{i}, i=1,2, \ldots, n$, such that $\sum_{i=1}^{n} \mho_{i}=1$ and the certain parameters $\emptyset_{i}=1, i=1,2, \ldots, n$, then the previous MONLP(II) takes the form

MONLP(III): $\min \quad \mho_{i}\left\{\left(h_{3}\right)\left(\frac{\vartheta_{i}-1}{\vartheta_{i}+1}\right)\right.$

$$
\begin{aligned}
& \times\left[b\left(\vartheta_{i}^{2 j-1}-1\right)+h_{4}\right] \\
& -2 \delta_{i 0}+\left(h_{2}\right)\left(\frac{\vartheta_{i}-1}{\vartheta_{i}+1}\right) \\
& \times\left[b\left(\vartheta_{i}^{j}-1\right)+h_{5}\right] \\
& \left.+b\left(\vartheta_{i}^{2 j-1}-1\right)\right\},
\end{aligned}
$$

subject to $b\left(\vartheta_{i}^{2 j-1}-1\right)$

$$
\begin{aligned}
& \leq\left(h_{1}-1\right)\left(\frac{\vartheta_{i}-1}{\vartheta_{i}+1}\right) \\
& \times\left[b\left(\vartheta_{i}^{j}-1\right)+h_{1}\right] \\
& \delta_{i 0}, \\
& b\left(\vartheta_{i}^{2 j}-1\right) \\
& \geq\left(h_{1}-1\right)\left(\frac{\vartheta_{i}-1}{\vartheta_{i}+1}\right) \\
& \times\left[b\left(\vartheta_{i}^{j}-1\right)+h_{1}\right] \\
&+\delta_{i 0}, \\
& 1-\vartheta_{i}<0, \quad \forall b=1,2, \ldots, \quad 1 \leq j \leq \frac{\mathscr{F}}{2} .
\end{aligned}
$$

From the Kuhn-Tucker conditions we have

$$
\begin{aligned}
& \frac{1}{2}\left\{\frac { 2 } { ( \vartheta _ { i } + 1 ) ^ { 2 } } \left[\left(h_{3}-1\right)\left[b\left(\vartheta_{i}^{2 j-1}-1\right)+h_{4}\right]\right.\right.\left.+\left(h_{2}-1\right)\left[b\left(\vartheta_{i}^{j}-1\right)+h_{5}\right]\right] \\
&+\left(\frac{\vartheta_{i}-1}{\vartheta_{i}+1}\right) b\left[\left(h_{3}-1\right)(2 j-1) \vartheta_{i}^{2 j-2}+\left(h_{2}-1\right) j \vartheta_{i}^{j-1}\right] \\
&\left.+b(2 j-1) \vartheta_{i}^{2 j-2}\right\} \\
&+u_{1}\left\{b(2 j-1) \vartheta_{i}^{2 j-2}-\frac{\left(h_{1}-1\right)}{\vartheta_{i}+1}\right. \\
&\left.\quad \times\left[\frac{2}{\vartheta_{i}+1}\left[b\left(\vartheta_{i}^{j}-1\right)+h_{1}\right]+\left(\vartheta_{i}-1\right) j b \vartheta_{i}^{j-1}\right]\right\} \\
&+u_{2}\left\{-b(2 j-1) \vartheta_{i}^{2 j-2}+\frac{\left(h_{1}-1\right)}{\vartheta_{i}+1}\right. \\
&\left.\quad \times\left[\frac{2}{\vartheta_{i}+1}\left[b\left(\vartheta_{i}^{j}-1\right)+h_{1}\right]+\left(\vartheta_{i}-1\right) j b \vartheta_{i}^{j-1}\right]\right\} \\
&-u_{3}=0, \quad u_{3}\left\{1-\vartheta_{i}\right\}=0 . \\
& u_{1}\left\{b\left(\vartheta_{i}^{2 j-1}-1\right)\left(\vartheta_{i}+1\right)-\left(h_{1}-1\right)\left(\vartheta_{i}-1\right)\right. \\
&\left.\times\left[b\left(\vartheta_{i}^{j-1}-1\right)+h_{1}\right]\right\}=0,
\end{aligned}
$$




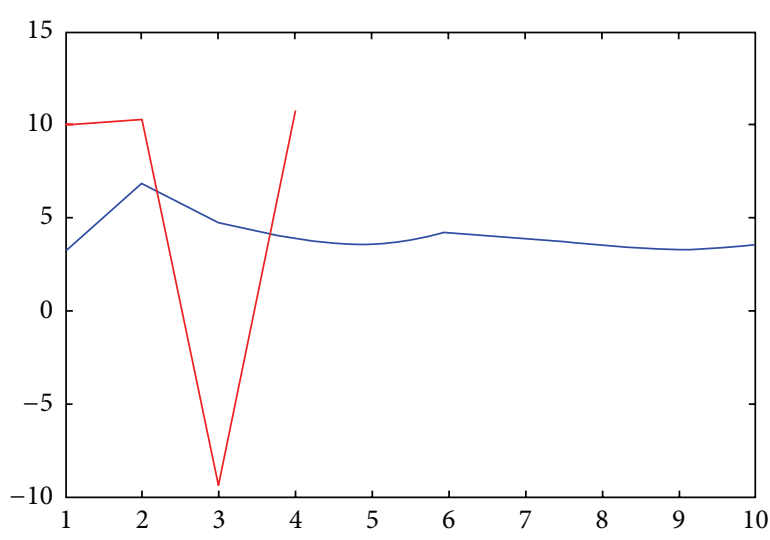

(a) $b=1$

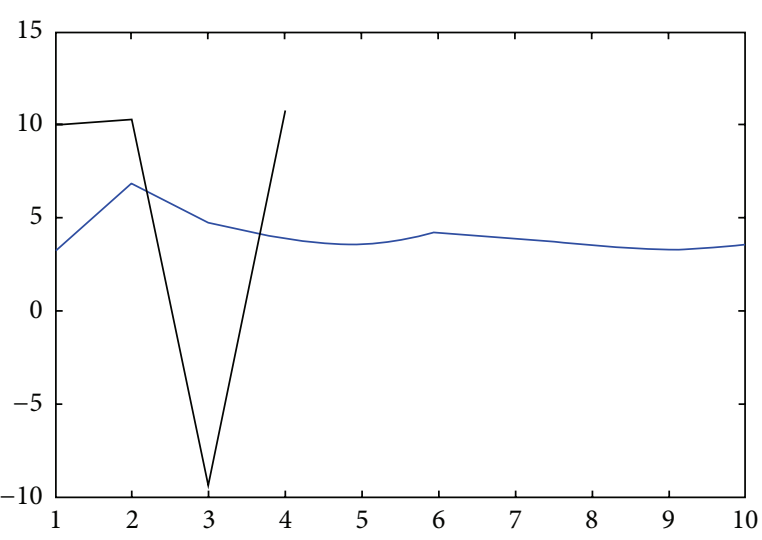

(b) $b=2$

Figure 2: (a) and (b) give the optimal expected value of the first meeting time between $S_{i}$ and the target that moves with discrete random walk in one dimension.

Solving (41)-(44), we found the optimal values of $\vartheta_{i}$ that give the optimal distances $H_{i j}$ for all $b=1,2, \ldots, 1 \leq j \leq$ $\mathscr{F} / 2$, and they are given by

$$
\vartheta_{i}=\sqrt{\frac{\widehat{\Upsilon}+\widehat{\Lambda}}{b(1-2 j) \vartheta_{i}^{2 j-2}}}-1, \quad \vartheta_{i}>1,
$$

where

$$
\begin{aligned}
\widehat{Y}=2[ & \left(h_{3}-1\right)\left[b\left(\vartheta_{i}^{2 j-1}-1\right)+h_{4}\right] \\
& \left.+\left(h_{2}-1\right)\left[b\left(\vartheta_{i}^{j}-1\right)+h_{5}\right]\right] \\
\widehat{\Lambda}= & b\left(h_{3}-1\right)(2 j-1) \vartheta_{i}^{2 j}-b\left(h_{3}-1\right)(2 j-1) \vartheta_{i}^{2 j-2} \\
& +b\left(h_{2}-1\right) j \vartheta_{i}^{j+1}-b\left(h_{2}-1\right) j \vartheta_{i}^{j-1} .
\end{aligned}
$$

\section{An Illustrative Example}

In this section, we clarify the effectiveness of this model by considering the following example.

Example 1. Suppose that a criminal drunk (target) leaves its cache and walks up and down through one of $n$-disjoint streets, totally disoriented. We model the streets as real lines with cache at the point $X_{0}$. In addition, assume that the criminal drunk takes unit steps, so we may record his position with an integer. Thus, for example, if he takes 5 steps to the left, he will be at a position $X_{0}-5$. If we consider the previous conditions in Theorems 2 and 3 hold, then the optimal expected value of the first meeting time depends on the optimal values of the distances $H_{i j}$ that the searcher $S_{i}$ should do it and the optimal values of $G_{i j}, j=2,4,6$. Using the constraint $G_{i(2 j-1)} \leq t \leq G_{i(2 j)}, i=1,2, \ldots, n, 1 \leq j \leq$ $\mathscr{F} / 2$, from Case 2 , such that $j=2,4,6$ and for $b=1,2$, in MONLP(III), and also using (38), we obtain the optimal values of $\vartheta_{i}$ that give the optimal values of $H_{i j}, G_{i j}$ in Table 1.

It is clear that the optimal distances $H_{i j}$ that $S_{i}$ should do it depend on $b$ where $\lambda_{i}=b \omega_{i}$ and $G_{i j}=\lambda_{i}\left(\vartheta_{i}^{j}-1\right)$.
TABLE 1: The optimal values of $\vartheta_{i}, H_{i j}$ and $G_{i j}, j=2,4,6, b=1,2$.

\begin{tabular}{ccccc}
\hline$b$ & $j$ & $\vartheta_{i}$ & $G_{i j}$ & $H_{i j}$ \\
\hline \multirow{3}{*}{1} & 2 & 1.8836 & 0.8836 & $0.27075+\delta_{i 0}$ \\
& 4 & 2.5265 & 1.5265 & $0.6607-\delta_{i 0}$ \\
& 6 & 2.6982 & 1.6982 & $0.7798+\delta_{i 0}$ \\
\hline \multirow{3}{*}{2} & 2 & 2.5226 & 3.0452 & $1.3162+\delta_{i 0}$ \\
& 4 & 2.7897 & 3.5794 & $2.2963-\delta_{i 0}$ \\
& 6 & 2.8652 & 3.7304 & $1.8+\delta_{i 0}$ \\
\hline
\end{tabular}

From the previous numerical calculations and by considering $\delta_{i 0}=10$, we get Figure 2 that shows the projection of the target's motion (one-dimensional random walk) on the $S_{i}$ path with $b=1,2$ where the current position on the line represented in the vertical axis versus the time steps at horizontal axis.

\section{Discussion and Conclusions}

A multiplicative generalized linear search plan for a onedimensional random walk target on one of $n$-disjoint real lines has been presented, where the target initial position is given by a random variable $X_{0}$. Therefore, the target will be meet if $E_{\tau_{\phi}}<\infty$, where $\phi(t)=\left(\phi_{1}(t), \phi_{2}(t), \ldots, \phi_{n}(t)\right)$ and $E_{\tau_{\phi}}$ is the expected value of the first meeting time between one of the searchers and the target. We discuss some properties that the search model should satisfy in Theorems 2 and 3. We introduce the proof of conditions that make a search plan finite in Theorem 4, based on the continuity of the search plan and the conditions in Theorems 2 and 3 to show that $E_{\tau_{\phi}}<\infty$. We provide more analysis by using Lemma 5, Theorems 2 and 3 in Theorem 6 to show that the search plan $\phi(t)$ is finite if the conditions ${ }^{2} \widehat{h}(x) \leq L(|x|),{ }^{2} \widehat{q}(x) \leq \widetilde{L}(|x|)$, if $\delta_{i 0}>0$ and ${ }^{1} \widehat{w}(x) \leq M(|x|),{ }^{1} \widehat{h}(x) \leq \widetilde{M}(|x|)$ if $\delta_{i 0}<0$, where $L(|x|)$, $\widetilde{L}(|x|), M(|x|)$, and $\widetilde{M}(|x|)$ are vectors of linear functions. We use Theorem 7 to show that if there exist a finite search plan then the expected value of the target initial position $E\left(\left|X_{0}\right|\right)$ is 
finite. It will also be interesting to see a direct consequence of Theorems 4, 6, and 7 satisfying the existence of a finite search plan if and only if $E\left(\left|X_{0}\right|\right)$ is finite. The existence of an optimal search plan has been proved in Theorem 10 .

To find the optimal distances $H_{i j}, i=1, \ldots, n, j=$ $1,2, \ldots$, that give the optimal search plan $\phi^{*}(t)=$ $\left(\phi_{1}^{*}(t), \phi_{2}^{*}(t), \ldots, \phi_{n}^{*}(t)\right)$ we solve the multiobjective nonlinear programming problem (MONLP) which contains the variables $\vartheta_{i}$ and the parameters $\lambda_{i}, i=1, \ldots, n$. We use the Kuhn-Tucker conditions to obtain the optimal values of $\vartheta_{i}$ that can give the optimal distances, after substituting (38), and then minimize the expected value of the first meeting time. The effectiveness of this model is illustrated using numerical example.

In future research, it seems that the proposed model will be extendible to the multiple searchers case by considering the combinations of movement of multiple targets.

\section{References}

[1] B. O. Koopman, "Search and Screening," OEG Report 56, The Summary Reports Group of the Columbia University Division of War Research (Available from the Center for Naval Analyses), 1946.

[2] S. J. Benkoski, M. G. Monticino, and J. R. Weisinger, "A survey of the search theory literature," Naval Research Logistics, vol. 38, pp. 469-494, 1991.

[3] J. R. Frost and L. D. Stone, "Review of Search Theory: Advances and Applications to Search and Rescue Decision Support," Final report CG-D-15-01, US Coast Guard Research and Development Center, 2001.

[4] B. J. MC Cabe, "Searching for one dimensional random walker," Journal of Applied Probability, vol. 11, pp. 86-93, 1974.

[5] A. A. Mohamed, "Generalized search for one dimensional random walker," International Journal of Pure and Applied Mathematics, vol. 19, pp. 375-387, 2005.

[6] A. B. El-Rayes and A. A. Mohamed, "Searching for a randomly moving target," in Proceedings of the 3rd ORMA Conference, vol. 2, pp. 323-329, Egypt, 1989.

[7] B. Fristedt and D. Heath, "Searching for a particl and on the real Line," Advances in Applied Probability, vol. 6, pp. 79-102, 1974.

[8] A. Ohsumi, "Optimal search for a markovian target," Naval Research Logistics, vol. 38, pp. 531-554, 1991.

[9] A. B. El-Rayes, A. E.-M. A. Mohamed, and H. M. Abou Gabal, "Linear search for a Brownian target motion," Acta Mathematica Scientia, vol. 23, no. 3, pp. 321-327, 2003.

[10] A. Mohamed, M. Kassem, and M. El-Hadidy, "Multiplicative linear search for a brownian target motion," Appli. Mathematical Model, vol. 35, pp. 4127-4139, 2011.

[11] A. Beck, "On the linear search problem," Israel Journal of Mathematics, vol. 2, pp. 221-228, 1964.

[12] A. Beck, "More on the linear search problem," Israel Journal of Mathematics, vol. 3, pp. 61-70, 1965.

[13] A. Beck and M. Beck, "Son of the linear search problem," Israel Journal of Mathematics, vol. 48, no. 2-3, pp. 109-122, 1984.

[14] A. Beck and M. Beck, "The linear search problem rides again," Israel Journal of Mathematics, vol. 53, no. 3, pp. 365-372, 1986.

[15] A. Beck and M. Beck, "Revenge of the linear search problem," SIAM Journal on Control and Optimization, vol. 30, no. 1, pp. 112-122, 1992.
[16] A. Beck and D. Newman, "Yet more on the linear search problem," Israel Journal of Mathematics, vol. 8, pp. 419-429, 1970.

[17] A. Beck and P. Warren, "The return of the linear search problem," Israel Journal of Mathematics, vol. 10, pp. 169-183, 1972.

[18] W. Franck, “On an optimal search problem," SIAM Review, vol. 7, pp. 503-512, 1965.

[19] P. Rousseeuw, "Optimal search paths for random variables," Journal of Computational and Applied Mathematics, vol. 9, pp. 279-286, 1983.

[20] D. J. Reyniers, "Coordinated search for an object hidden on the line," European Journal of Operational Research, vol. 95, no. 3, pp. 663-670, 1996.

[21] D. J. Reyniers, "Co-ordinating two searchers for an object hidden on an interval," Journal of the Operational Research Society, vol. 46, no. 11, pp. 1386-1392, 1995.

[22] Z. T. Balkhi, “The generalized linear search problem, existence of optimal search paths," Journal of the Operations Research Society of Japan, vol. 30, pp. 399-420, 1987.

[23] Z. T. Balkhi, "Generalized optimal search paths for continuous univariate random variable," Operations Research, vol. 23, pp. 67-96, 1987.

[24] A. A. Mohamed, H. M. Abou Gabal, and M. A. El-Hadidy, "Coordinated search for a randomly located target on the plane," European Journal of Pure and Applied Mathematics, vol. 2, pp. 97-111, 2009.

[25] A. A. Mohamed, H. A. Fergany, and M. A. El-Hadidy, "On the coordinated search problem on the plane," Istanbul University Journal of the School of Business Administration, vol. 41, pp. 80102, 2012

[26] A. Mohamed and M. El-Hadidy, "Existence of a periodic search strategy for a parabolic spiral target motion in the plane," Afrika Matematika, vol. 24, no. 2, pp. 145-160, 2013.

[27] A. Mohamed and M. El-Hadidy, "Coordinated search for a conditionally deterministic target motion in the plane," European Journal of Mathematical Sciences, vol. 2, no. 3, pp. 272-295, 2013.

[28] W. Feller, An Introduction to Probability Theory and Its Applications, Wiley, New York, NY, USA, 2nd edition, 1966. 


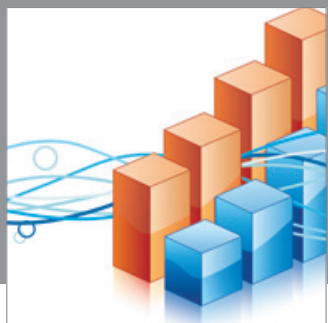

Advances in

Operations Research

mansans

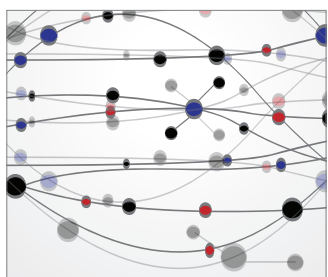

The Scientific World Journal
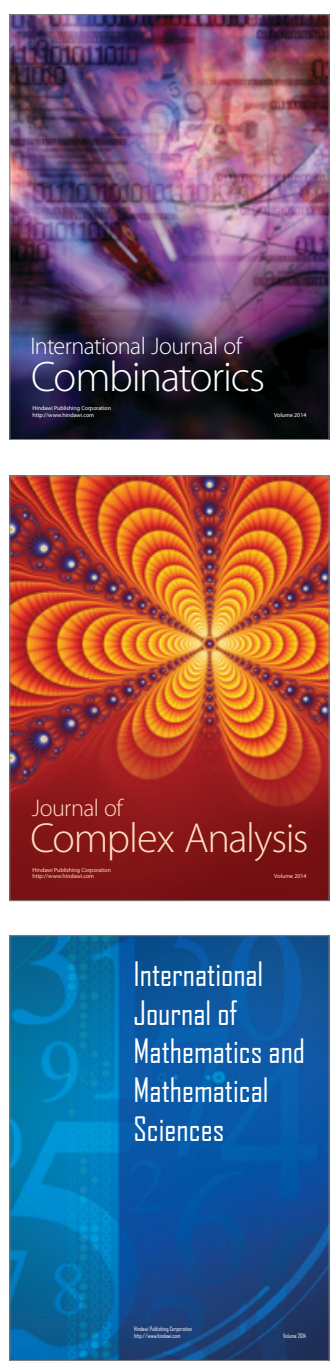
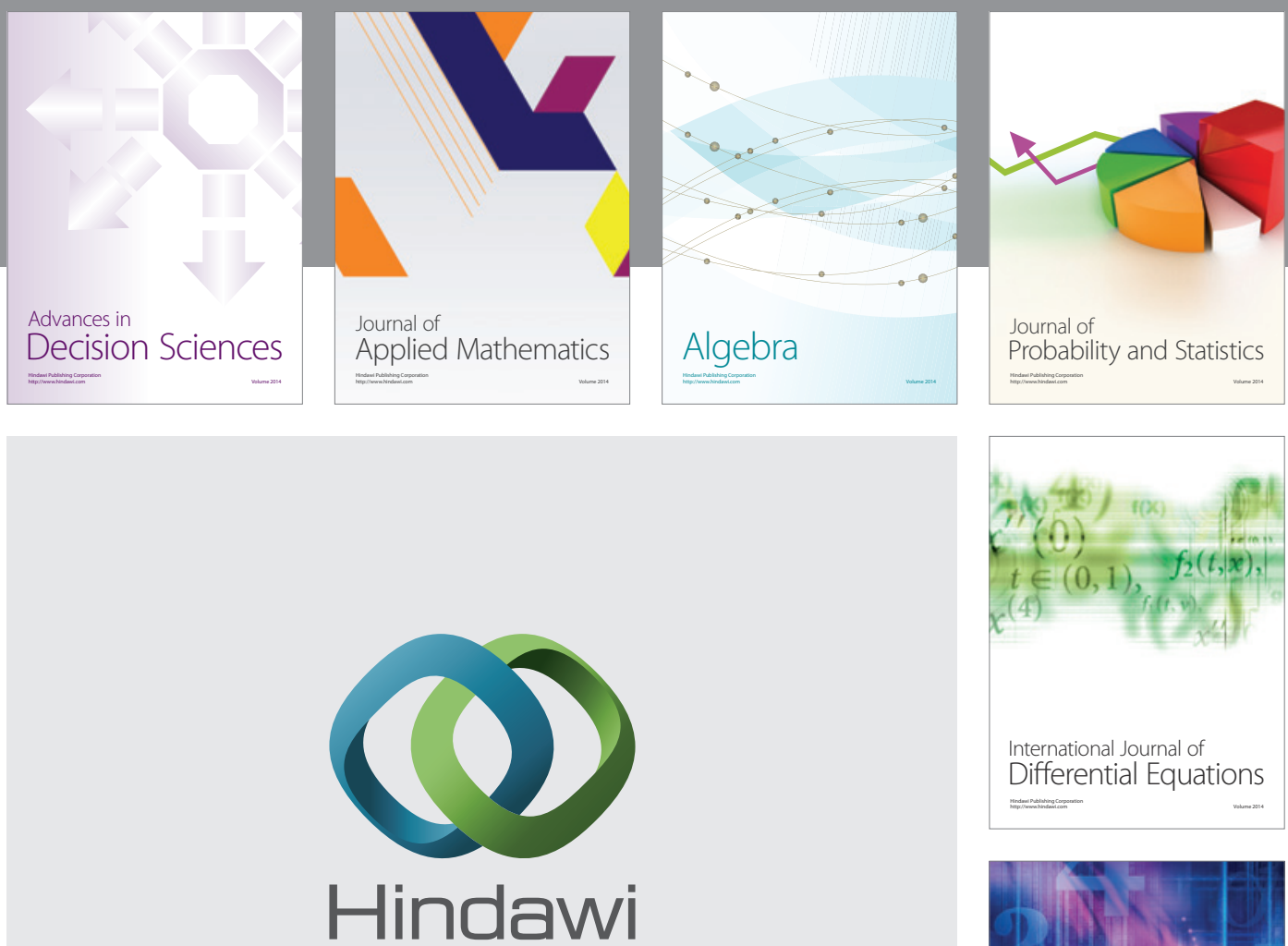

Submit your manuscripts at http://www.hindawi.com
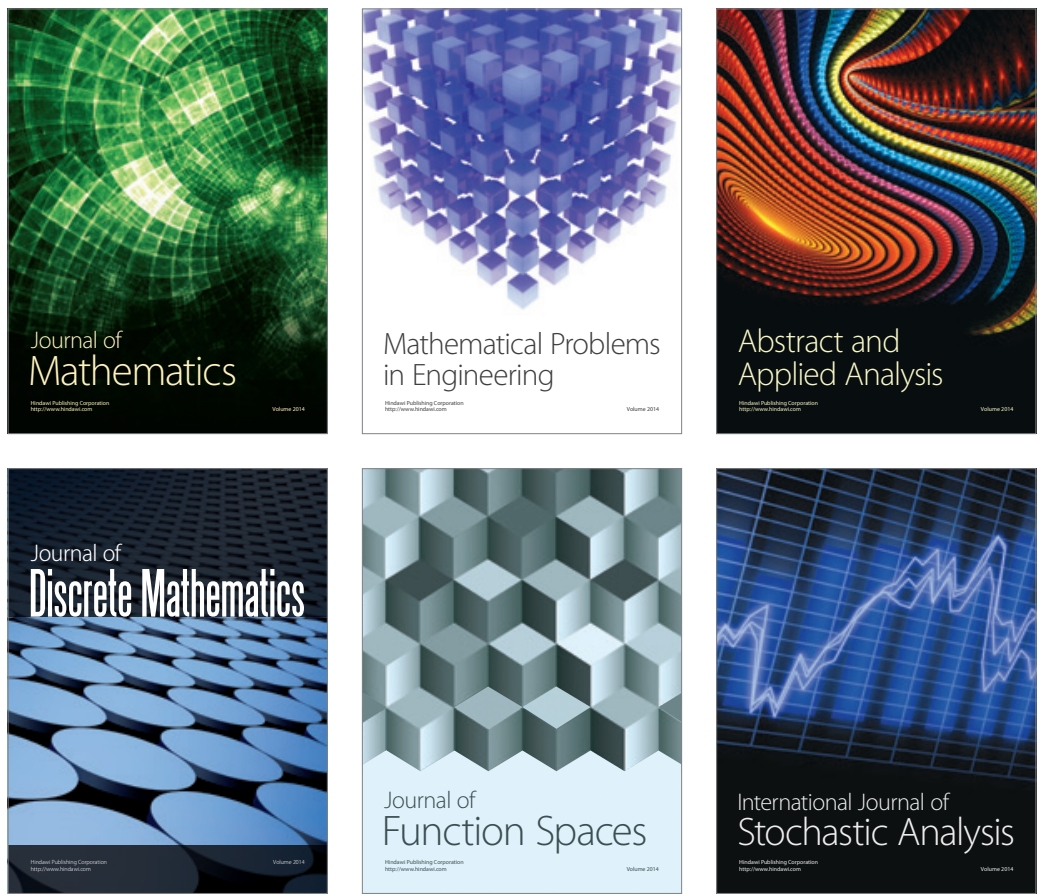

Journal of

Function Spaces

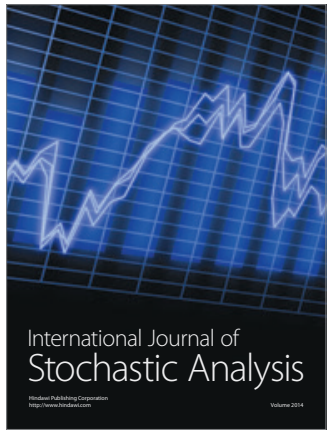

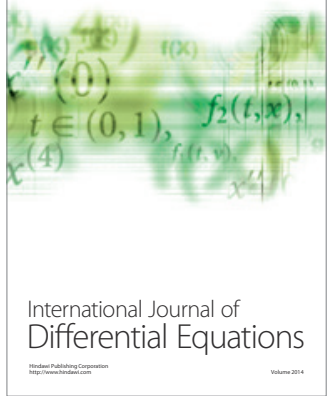
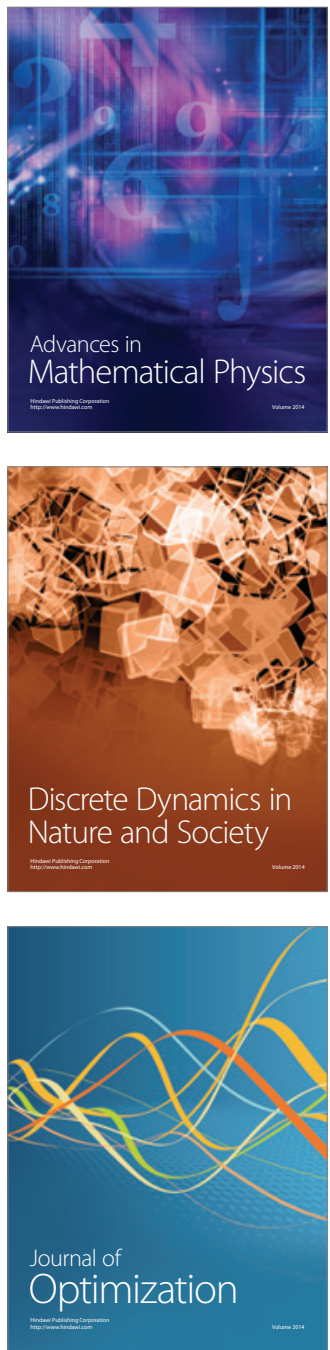